\title{
Engineered Porous/Hollow Burkholderia Pseudomallei Loading Tumor Lysate as a Vaccine
}

\section{Feng-Ying Huang}

Hainan Medical University

Shu-Zhen Dai

Hainan Medical University

Jin-Yan Wang

Hainan Medical University

Ying-Ying Lin

Hainan Medical University

Cai-Chun Wang

Hainan Medical University

Wu-Ping Zheng

Hainan Medical University

Guang-hong Tan ( $\square$ tanhoho@163.com )

Hainan Medical University https://orcid.org/0000-0002-6406-0331

Article

Keywords: Tumor vaccine, Burkholderia pseudomallei, Vaccine carrier, Immunosuppressive tumor microenvironment, Ferroptosis

Posted Date: June 10th, 2021

DOI: https://doi.org/10.21203/rs.3.rs-579203/v1

License: (c) (i) This work is licensed under a Creative Commons Attribution 4.0 International License.

Read Full License 


\section{Abstract}

Due to its size, shape, and inherent expression of pathogen-associated molecular patterns and invasionassistant adhesion proteins, Burkholderia pseudomallei can easily attach to, and then be internalized by, dendritic cells (DCs), leading to more efficient antigen cross-presentation if modified as carrier. Herein, we engineered Burkholderia pseudomallei as a porous/hollow carrier (SB) for loading tumor lysates $(\mathrm{L})$ and CpG (C) to be used as a tumor vaccine (SB-LC). We found that the adhesion proteins of Burkholderia pseudomallei promote internalization of the SB-LC vaccine by DCs, and result in enhanced DC maturation and antigen cross-presentation. SB-LC induces robust cellular and humoral antitumor responses that synergistically inhibit tumor growth with minimal adverse side effects in several tumor models. Moreover, SB-LC vaccination reverses the immunosuppressive tumor microenvironment, apparently as a result of $\mathrm{CD} 8^{+}$-induced tumor ferroptosis. Thus, SB-LC is a potential model tumor vaccine for translating into a clinically viable treatment option.

\section{Introduction}

Currently, the most effective vaccines are those that are related to pathogenic microorganisms. Vaccination with such vaccines has led to the effective control or even complete eradication of many infectious diseases, such as tuberculosis, smallpox, measles, poliomyelitis, tetanus, and diphtheria, because the host immune system can evoke a stronger response against the pathogenic microorganisms

1 . The success of these vaccines can be attributed to the strong immunogenicity of the pathogenic microorganisms as a result of their expression of pathogen-associated molecular patterns (PAMPs) ${ }^{2,3}$. Due to the conserved molecular structures of PAMPs, the host immune system can rapidly distinguish PAMPs as "non-self" and activate a specific adaptive immune response ${ }^{4}$. Thus, PAMPs have been widely used as adjuvants to enhance immune responses during vaccination ${ }^{5}$. In addition, the specific physical properties of the microorganisms are also known to influence the host immune response. A previous study showed that rod-like particles of $2-3 \mu \mathrm{m}$ are recognized and internalized by macrophages ${ }^{6,7}$. A recent study also showed that a biomimetically-engineered Bacillus could potentiate vaccination against tumors $^{8}$. These findings indicate that rod-shapedbacteriacan be modified as carriers for loading tumor antigen and/or adjuvants as novel model vaccines.

Cross-presentation is the capability of antigen-presenting cells (APCs) to take up, process, and present extracellular antigens with $\mathrm{MHC}$ I molecules to $\mathrm{CD} 8^{+} \mathrm{T}$ cells. Cross-presentation is of particular importance for the development of cancer immunotherapy vaccines, because it can stimulate naive cytotoxic $\mathrm{CD}^{+}{ }^{+} \mathrm{T}$ cells into activated cytotoxic $\mathrm{CD} 8^{+} \mathrm{T}_{\text {cells }}{ }^{9,10}$. Burkholderia pseudomallei (B. pseudomallei)is a Gram-negative rod-shaped bacterium ${ }^{11}$. As an intracellular bacterium, B. pseudomallei has developed various adhesion proteins, such as IV pilus protein PilA, BoaA, and BoaB. Most of these adhesion proteins are specific ligands for protease-activated receptor-1 (PAR1), which is inherently expressed on the surface of dendritic cells (DCs), macrophages, endothelial cells, and platelets ${ }^{12,13}$. Once bound to the host cells, the bacteria enter through endocytosis ${ }^{12,13}$. Therefore, we hypothesized 
that using engineered porous/hollow B. pseudomallei as carriers may be superior to other rod-shaped bacteria for loading tumor antigen and/or adjuvants as vaccines.

DC vaccines pulsed with tumor lysates have been reported as effective vaccines for the treatment of tumors ${ }^{14,15,16}$. Therefore, in this study, we engineered $B$. pseudomallei as a porous/hollow carrier (SB) for loading tumor lysates $(\mathrm{L})$ and $\mathrm{CpG}(\mathrm{C})$ as a tumor vaccine (SB-LC), and investigated its antitumor effects in murine models.

\section{Results And Discussion}

\section{Fabrication of the porous/hollow B. pseudomallei loading tumor lysates and CpG}

Fabrication of the porous/hollow B. pseudomallei was performed as shown in Figure 1A. Given that a part of the bacteria was removed and only the backbones of the bacteria were kept, we referred this modified bacteria as semi-B. pseudomallei (SB). To testify weather SBs were successfully constructed and loaded with tumor lysates and $\mathrm{CpG}$, SBs, OVA-B16 tumor lysate, and $\mathrm{CpG}$ were labeled with FITC, rhodamine, and DAPI, respectively, and then observed by a high-resolution confocal laser scanning microscope. The resultant images show that the OVA-B16 tumor lysate and CpG were co-localized inside the SBs (Figure 1B), suggesting successful preparation of SB-loading of OVA-B16 tumor lysates and CpG. SBs loaded with $\mathrm{CpG}$ and other tumor lysates, such as CT26, 4T1, and H22 were also prepared (Figures S1A-S1C). The co-localization of SBs, tumor lysates, and $\mathrm{CpG}$ indicates the successful loading of tumor lysates and $\mathrm{CpG}$ into SBs, and confirms the porous/hollow structure of SBs.

\section{SBs loading with tumor lysate and CpG promotes dendritic cell (DC) maturation and antigen cross-presentation}

Cross-presentation of extracellular tumor antigens by APCs, especially by DCs, to CD $8^{+} \mathrm{T}$ cells is particularly important for the development of tumor vaccines, because it can stimulate naive cytotoxic $\mathrm{CD}^{+} \mathrm{T}$ cells into activated cytotoxic $\mathrm{CD} 8^{+} \mathrm{T}$ lymphocytes (CTLs) ${ }^{9,10}$. Thus, successful tumor vaccines are often designed to efficiently stimulate cross-presentation of tumor antigens by DCs so as to elicit a sufficient CTL response ${ }^{17}$. To detect whether SBs loaded with OVA-B16 tumor lysates and CpG (SB-LC) could augment $D C$ maturation and activation, mouse bone-marrow-derived dendritic cells (BMDCs) were co-cultured with OVA-B16 lysate pulsed with CpG (LC), SB loaded with CpG (SB-C), SB loaded with OVAB16 lysate (SB-L), and SB-LC for $24 \mathrm{~h}$. The maturation markers (CD86, CD80, and CD40) on BMDCs and activation cytokines (TNF-a, IFN- $y$, and IL-6) in the supernatants were detected by flow cytometry and ELISA, respectively. BMDCs co-cultured with SB-C, SB-L, and SB-LC expressed more CD86 compared to LC (Figures 1C and S1D), CD80 (Figures 1D and S1E), and CD40 (Figures 1E and S1F). The highest expression of these markers was found on the SB-LC-co-cultured BMDCs (Figures 1C-1E and S1D-S1F). 
Consistently, BMDCs co-cultured with SB-C, SB-L, and SB-LC secreted more TNF-a (Figure 1F), IFN-y (Figure 1G), and IL-6 (Figure 1H) than those co-cultured with SB-LC; indeed, SB-LC-co-cultured BMDCs produced the highest levels of cytokines of all groups tested (Figures $1 \mathrm{~F}-1 \mathrm{H}$ ). These data indicate that the engineered SB facilitates the activation and maturation of BMDCs.

To evaluate whether SB-LC vaccination could facilitate cross-presentation, SB-LC loaded with OVA-B16 lysate that harboured OVA SIINFEKL peptide was co-cultured with BMDCs, and the SIINFEKL-MHC I complexes and MHC II molecules were detected by flow cytometry. Significant up-regulation of the SIINFEKL-MHC I complex was found on the SB-LC-co-cultured BMDCs (Figures 1I and S1G). Similarly, the expression of MHC II molecules was also significantly increased in the SB-LC-co-cultured BMDCs (Figures $1 \mathrm{~J}$ and $\mathrm{S} 1 \mathrm{H})$. These data strongly suggest that SB-LC promotes tumor antigen cross-presentation by BMDCs.

\section{Adhesion proteins of $B$. pseudomallei on SBs facilitate the internalization of tumor antigens}

Internalization of extracellular tumor antigens by DCs is a necessary step that influences the efficiency of cross-presentation of tumor antigens. Besides, the most commonly known mannose receptor (MR) agonist, $B$. pseudomallei, also possesses a set of invasion-assisting apparatus, including adhesion proteins, which are ligands for PAR1 inherently expressed on the surface of DCs ${ }^{12,13}$. In addition, a rodshaped Bacillus that keeps its intrinsic MR agonist has been reported as an excellent carrier for use in the development of tumor vaccines ${ }^{8}$. Therefore, we hypothesized that the engineered SB may be superior to other rod-shaped-bacterium carriers if it were to retain its intrinsic MR agonist and adhesion proteins. To validate this hypothesis, we prepared a semi-Escherichia coli (SEs) and compared its uptake by BMDCs with that of SB. To this end, we co-cultured BMDCs with comparable FITC-labeled SBs or SEs, and then detected the efficiency of endocytosis by flow cytometry. As predicted, SBs were more easily internalized by BMDCs than SEs (Figure 2A). In addition, we blocked PAR1 and MR alone or in combination with monoclonal antibodies, and then compared the endocytosis efficiency of SBs and SEs by BMDCs. Blockade of MR alone led to significantly decreased uptake of both SBs and SEs, but blockage of PAR1 only influenced SB uptake (Figure 2A). Moreover, combined blockage of PAR1 and MR resulted in significantly decreased uptake of both SBs and SEs to similar levels (Figure 2A). These data suggest both PAR1 and MR pathways are involved in the uptake of SBs, and that the uptake of SBs by BMDCs was easier than the uptake of SEs.

Phagocytosis and micropinocytosis are the main routes by which exogenous antigens enter APCs with the assistance of actin polymerization $18,19,20$. Once bound to the host adhesion proteins, $B$. pseudomallei induces polymerization of host actin, which facilitates internalization into DCs ${ }^{12}$. Therefore, we blocked the actin polymerization by cytochalasin D (Cyt D) and compared the endocytosis efficiency of SBs and SEs by BMDCs. The results showed that the increased internalization of SBs by BMDCs was significantly suppressed to a similar level to SEs after Cyt D treatment (Figure 2A), indicating 
the involvement of actin polymerization in this process. In addition, B16 lysate and CpG loading in the LC, SB-C, SB-L, and SB-LC were labeled with FITC and DAPI, respectively, and then co-cultured with BMDCs and analysed by flow cytometry. Consistently, all of the SB formulas (SB-L and SB-LC) loaded with FITClabeled B16 lysate (Figure 2B) or DAPI-labeled CpG (Figure 2C) revealed higher internalization into BMDCs. Taken together, these findings suggest that the adhesion proteins on the SBs facilitate uptake of the loading tumor antigens through actin-polymerization-dependent phagocytosis and/or micropinocytosis.

\section{SBs loaded with tumor lysates promote antigen cross- presentation via both cytosolic and vacuolar pathways}

Two pathways, namely cytosolic and vacuolar, are considered to be related to cross-presentation of exogenous antigens ${ }^{10,21}$. Both the vacuolar and cytosolic diversion pathways are initiated through the endocytosis of an extracellular antigen by APCs ${ }^{10,22}$. The key difference between the cytosolic and vacuolar pathways is that the cytosolic pathway is sensitive to proteasome inhibitors ${ }^{10,21}$. To determine whether SBs could promote antigen cross-presentation, and its pathways, we observed whether there was co-localization of FITC-labeled tumor lysates with EEA1 (an endosome marker) or with lysosome (marked by LysoTracker Red). Compared with SB-co-cultured BMDCs, BMDCs co-cultured with SB-L or SB-LC showed obvious co-localization of tumor lysates with EEA1 (Figure 2D) and lysosomes (Figure 2E). In addition, flow cytometry detection of SIINFEKL-MHC I complexes showed that MG132 (a proteasome inhibitor; Figure 3F) or leupeptin (Leup, a lysosome protease inhibitor; Figure $2 \mathrm{G}$ ) blockage significantly neutralized the increased expression of SIINFEKL-MHC I complexes on the BMDCs co-cultured with SB-L or SB-LC (Figure 2F and 2G). However, combination blockage with both MG132 and Leup resulted in much stronger inhibition of SIINFEKL-MHC I complex expression (Figure 2H). These findings, together with the data shown in Figures 1I and S1G, indicate that SBs loaded with tumor lysates and CpG can promote antigen cross-presentation via both cytosolic and vacuolar pathways.

\section{SB-LC vaccination induces therapeutic and prophylactic anti-tumor responses, with no obvious side effects}

Therapeutic and prophylactic anti-tumor responses were first observed in OVA-B16 and CT26 murine models. To observe the therapeutic anti-tumor activities, mice were subcutaneously injected with tumor cells and then vaccinated with SB-LC, SB-L, SB-C, or LC twice on days 3 and 10 when the tumor masses were palpable. Compared with SB-C, vaccination with LC, SB-L, and SB-LC obviously delayed tumor growth and prolonged survival, and more significant tumor suppression and longer survival were found in the mice vaccinated with SB-LC (Figures 3A, 3B, and S2A). To observe prophylactic antitumor effects, the mice were first vaccinated with SB-LC, SB-L, SB-C, or LC on days 0 and 7, and the tumor models were established on day 7. The results were similar to those of the therapeutic analysis (Figures $3 \mathrm{C}, \mathrm{S} 2 \mathrm{~B}$ and 
$\mathrm{S} 2 \mathrm{C})$. Anti-metastatic activities were observed in the prophylactic protocol in 4T1-bearing mice. Compared with the SB-C, vaccination with LC, SB-L, and SB-LC obviously increased the suppression of tumor metastasis with the best in the SB-LC vaccination (Figure 3D).

In this study, we also considered the side effects induced by various formulations in normal female C57BL/ 6 and BALB/C mice. We vaccinated these mice with LC, SB-C, SB-L, or SB-LC but without injection of tumor cells. On day 180 , the major organs and serum were collected for $\mathrm{H} \& \mathrm{E}$ and biochemical analysis. None of the mice showed any differences in gross measures such as life span, ruffling of fur, and behaviour. Body weight and fertility were also observed as indirect indicators of side effects. Our experiments revealed no impact on the body weight (Figure S3A) and fertility (Figure S3B). Additionally, the parturition day and the number of pups born also showed no significant difference among the mice vaccinated with LC, SB-C, SB-L, and SB-LC (Figure S3B). No pathologic changes in the heart, liver, spleen, lung, and kidney were found (Figure S3C). Moreover, the white blood cell and red blood cell counts were examined as measures of bone-marrow toxicity, creatinine level as a measure of kidney toxicity, aspartate transaminase and alanine transaminase as measures of liver toxicity, and lactic dehydrogenase as a measure of multi-organ toxicity. None of these parameters were significantly different among mice vaccinated with LC, SB-C, SB-L, and SB-LC (Figure S3D).

\section{SB-LC vaccination provokes tumor-specific cellular and humoral immunity}

Cellular immunity, especially via CTLs, is the major weapon against tumors. Therefore, we evaluated whether splenocytes could bind to OVA257-264-bound H-2 $\mathrm{K}^{\mathrm{b}}$ tetramers. Vaccination with either SB-L or SB-LC significantly increased the number of OVA tetramers on $\mathrm{CD} 8^{+}$splenocytes, with the most significant increase observed on the SB-LC-vaccinated lymphocytes (Figures 4A and 4B). Specific CTL responses were also detected using splenocytes as effector cells and B16 or CT26 cells as target cells. Consistently, target cell lysis was significantly induced by SB-LC-vaccinated lymphocytes (Figures 4C and 4D). In this study, we adapted an in vivo method to detect specific CTL responses by injecting CFSElabeled $\mathrm{H} 22$ cells into the abdominal cavity and observed the cell proliferation by flow cytometry. The number of $\mathrm{CD}^{4} 5^{-} \mathrm{CFSE}-l a b e l e d \mathrm{H} 22$ cells were significantly increased and proliferated for approximately three generations in unvaccinated normal mice; however, this was markedly hindered in LC-, SB-C-, and SB-LC-vaccinated mice with the most in the SB-LC-vaccinated mice (Figure 4E). Additionally, the number of $\mathrm{CD} 45^{-}$CFSE-labeled $\mathrm{H} 22$ cells was used to calculate the specific cytotoxicity. As predicted, the most cytotoxicity was observed in the SB-LC-vaccinated mice (Figure 4F). Moreover, the CD8 ${ }^{+}$splenocytes from the SB-LC-vaccinated mice secreted more active cytokines, such as IFN-y (Figure 4G), TNF-a (Figure 4H), and IL-2 (Figure 4I). These data indicate that SB-LC formulation enhances the production of tumorspecific cellular immunity.

We found SB-LC could also enhance tumor-specific humoral immunity. In western-blot analysis, sera from the SB-L- and SB-LC-vaccinated mice had high titres of IgG (Figure 4J). These were further confirmed by 
ELISPOT assay, in which the greatest number of monocytes secreting tumor-specific IgG was observed in the SB-LC-vaccinated mouse spleens (Figures 4K and 4L). ELISA data also showed similar results (Figure 4M). In addition, the titres of IgG1 and IgG2a were significantly increased in SB-LC- and SB-L-vaccinated mice (Figure $4 \mathrm{~N}$ ). These data suggest that SB-LC vaccination enhances humoral immunity by stimulating both Th1 and Th2 immune responses.

\section{Cellular and humoral immunities synergistically inhibit tumor growth}

To observe whether cellular and humoral immunity is directly related to the antitumor activities, splenocytes and antibodies were isolated from B16 or CT26 mice vaccinated with different formulations, and adoptively transferred into syngeneic tumor-bearing mice. Figure $5 \mathrm{~A}$ shows the images of the tumor masses on day 15 . Tumour growth was obviously decreased in the mice that received splenocytes from the SB-LC-vaccinated mice (Figure 5B). In addition, transfusion of antibodies from vaccinated mice into B16-bearing syngeneic mice significantly suppressed tumor growth (Figure $5 \mathrm{C}$ ). These data suggest that both cellular and humoral immunities induced by SB-LC are involved in the anti-tumor activities.

In this study, we investigated whether the cellular and antibody immune responses were synergized to combat tumor growth ${ }^{23}$. Splenocytes and antibodies isolated from H22 SB-LC-vaccinated mice were used to perform this study. Twenty BALB/c mice were injected with CFSE-labeled $\mathrm{H} 22$ cells, randomly divided into four groups, and then intraperitoneally injected with phosphate-buffered saline (PBS), splenocytes, and antibodies alone or combination. Injection of splenocytes or antibodies alone markedly suppressed $\mathrm{H} 22$ cell proliferation. However, the greatest suppression was observed in the combination mice (Figure 5D), in which the synergistic index was 1.448 (Figure 5E), suggesting that the cellular and antibody immune responses induced by SB-LC vaccination synergistically inhibit tumor growth.

To investigate the role of $\mathrm{CD} 8^{+}$and $\mathrm{CD} 4^{+} \mathrm{T}$ cells in the induction of antitumor immunity, $\mathrm{CD} 8^{+}$and $\mathrm{CD} 4^{+} \mathrm{T}$ cells of tumor-bearing mice were depleted by intraperitoneal injection of anti-CD4 or anti-CD8 monoclonal antibodies, and then the tumor volumes and titres of tumor-specific IgG were recorded on day 15 . To simplify the experiment, we only observed tumor-bearing mice vaccinated with SB-LC and SB-C. Depletion of $\mathrm{CD}^{+}$and $\mathrm{CD} 4^{+} \mathrm{T}$ cells alone led to significant tumor growth in SB-LC-vaccinated mice, but more obvious tumor growth was found in the $\mathrm{CD} 8^{+}$-depleted mice (Figure $5 \mathrm{~F}$ ). Consistently, depletion of CD $4^{+}$ but not $C D 8^{+} T$ cells resulted in a decreased titre of tumor-specific IgG in the SB-LC-vaccinated mice (Figure $5 \mathrm{G}$ ). These results suggest that both $\mathrm{CD} 8^{+}$and $\mathrm{CD} 4^{+} \mathrm{T}$ lymphocytes are involved in the induction of an antitumor immune response by SB-LC vaccination, but that $\mathrm{CD} 8^{+} \mathrm{T}$ cells play a more important role in the process. 


\section{SB-LC vaccination refreshes the immunosuppressive tumor microenvironment}

In the immunosuppressive tumor microenvironment, tumor cells recruit and expand various types of inhibitory tumor-infiltrating lymphocytes (TILs), including regulatory T cells (Tregs), myeloid-derived suppressor cells (MDSCs), tolerogenic DCs, and M2-type tumor-associated macrophages (TAMs) 17, 24, 25, 26. To investigate whether SB-LC vaccination could harness this immunosuppressive microenvironment, we observed the in situ infiltration of CD8 ${ }^{+}$lymphocytes, MDSCs, Tregs, and M2 TAMs in mouse tumor tissues. Significantly more $\mathrm{CD} 8^{+}$cells were found in SB-LC-vaccinated tissues compared to the other three groups (Figure 6A). By contrast, MDSCs and Tregs were rarely found in the SB-LC-vaccinated tumor tissues (Figure 6A). We also performed flow cytometry analysis of the cells in B16 and CT26 tumor tissues. The populations of $\mathrm{CD} 45^{-}$tumor cells and $\mathrm{CD} 45^{+} \mathrm{CD} 4^{+}$and $\mathrm{CD} 45^{+} \mathrm{CD} 11 \mathrm{~b}^{+}$lymphocytes were gated out from the total cell population (Figure S4). We then further analysed whether these lymphocytes were IFN- $\gamma$-secreting CD8, MDSCs, Tregs, or TAMs. The results showed that the percentage of IFN- $\gamma$ secreting $\mathrm{CD}^{+}$lymphocytes was significantly increased in the SB-LC-vaccinated tumor tissues (Figure 6B and S4A). However, the percentage of MDSCs (Figure 6C and S4B), Tregs (Figures 6D and S4C), and M2 TAMs (Figures 6E and S4D) was significantly decreased in the SB-LC-vaccinated tumor tissues. These results suggest that the immunosuppressive microenvironment is effectively reversed by SB-LC vaccination.

\section{CTL-induced tumor ferroptosis reverses the immunosuppressive tumor microenvironment}

Ferroptosis is a form of regulated cell death that differs from apoptosis in that it is induced by extra irondependent accumulation of lipid peroxide ${ }^{27}$. Several lines of evidence show that ferroptosis may be involved in a variety of pathological scenarios ${ }^{28,29}$. Indeed, a recent study has linked cancer immunotherapy by programmed death-ligand 1 blockade to ferroptosis, during which $\mathrm{CD} 8^{+} \mathrm{T}$ cells induce tumor ferroptosis that is beneficial for the suppression of tumor growth ${ }^{30}$. In addition, evidence indicates that dying cells, such as apoptotic cells, are able to recruit various immune cells into tumor tissues by secreting "find me" and "eat me" signals, resulting in the modulation of tumor microenvironment ${ }^{31}$. At present, whether ferroptotic cells release these signals is not fully understood. In this study, we considered that ferroptotic cells can also release these signals and can therefore also modulate the immunosuppressive tumor microenvironment. To verify this hypothesis, we sought to determine whether SB-LC vaccination could induce ferroptosis in B16- and CT26-bearing mice. SB-LC vaccination resulted in obviously increased lipid reactive oxygen species (ROS) in CD45-tumor cells (Figures 7A, S5A and S5B), but not in $\mathrm{CD} 45^{+}$cells (Figures S5A and S5B), and also decreased tumor growth (Figures 7B, S5C and S5D). The increased lipid ROS and decreased tumor growth induced by SB-LC vaccination were neutralized by co-treatment with the ferroptosis inhibitor liproxstatin-1 (Figures 7A, 7B and S5A-S5D). In 
addition, co-culture of these tumor cells with $\mathrm{CD} 8^{+} \mathrm{T}$ lymphocytes from the SB-LC-vaccinated mice significantly augmented lipid ROS (Figures 7C, S5E and S5F) and increased RSL3-induced cell death (Figures 7D, S6A and S6B). These effects were partially reversed by ferrostatin-1 (Figures 7D, S6A and $\mathrm{S} 6 \mathrm{~B})$. These data indicate that $\mathrm{CD} 8^{+} \mathrm{T}$ lymphocytes activated by SB-LC vaccination can induce tumor cell ferroptosis.

To investigate whether ferroptosis induced by SB-LC vaccination was involved in the reversal of the immunosuppressive tumor microenvironment, we blocked ferroptosis by liproxstatin-1. Compared with the SB-C-vaccinated tumor tissues, the increased IFN-y-secreting $\mathrm{CD} 8^{+}$cells induced by SB-LC vaccination were significantly attenuated by liproxstatin-1 treatment (Figures 7E and S7A). However, the significantly decreased percentage of MDSCs (Figures 7F and S7B), Tregs (Figures 7G and S7C), and M2 TAMs (Figures $7 \mathrm{H}$ and S7D) were rescued to similar levels to those induced by SB-C vaccination combined with liproxstatin-1 treatment (Figures 7F-7H and 7B-7D). These data suggest that ferroptosis induced by B$L C$ vaccination is involved in reversing the immunosuppressive tumor microenvironment.

\section{Materials And Methods}

\section{Fabrication of semi-Burkholderia Pseudomallei, loading tumor lysates and CpG}

Semi-B. Pseudomallei (SB) loading tumor lysates and CpG were constructed somewhat as previously reported but without hydrothermal process which was replaced by a deeply dialysis and then an electrophoretic process ${ }^{8}$. In brief, B. pseudomallei (strain BPC006) ${ }^{32}$ was first attenuated by ionizing radiation at $10 \mathrm{~Gy}$ (RS-2000-PRO; Rad Source, GA, USA), and then treated with 95\% ethyl alcohol for $1 \mathrm{~h}$ following another treatment by $1.5 \%(\mathrm{v} / \mathrm{v})$ Triton $\mathrm{X}-100$ for $5 \mathrm{~h}$. After that, the bacteria were put in a 0.22 $\mu \mathrm{m}$ poly tetra fluoroethylene (PTFE) membrane filter (Jinteng, Shanghai, China) and then dialysed in ultrapure water at $4^{\circ} \mathrm{C}$ for $5 \mathrm{~h}$. After that, the membrane filter was merged in the electrophoresis tank of Mini Transblot device (Bio-Rad, Hercules, CA) containing Tris-glycine-SDS buffer (T7777, Sigma-Aldrich), and then electrophoresis was performed by $100 \mathrm{~V}$ for $2 \mathrm{~h}$ at $4^{\circ} \mathrm{C}$. After that, the bacteria were washed twice with ultrapure water. The tumor lysates used in this study were prepared as previously reported ${ }^{33}$, and CpG1826 (5'-TCCATGACGTTCCTGACGTT-3') was purchased from TAKARA (Dalian, China). To load the SBs with CpG (SB-C), tumor lysates (SB-L), a combination of tumor lysates and CpG (SB-LC), tumor lysates, or $\mathrm{CpG}$, were subjected to vacuum negative pressure and then successively incubated with $50 \mu \mathrm{g}$ SBs in $100 \mu \mathrm{l} \mathrm{PBS}$ at $4^{\circ} \mathrm{C}$ for $12 \mathrm{~h}$. The free tumor lysates or $\mathrm{CpG}$ were washed with PBS by three centrifugations. The loading efficiency of tumor lysates or $\mathrm{CpG}$ was determined from the difference in mass between the initial incubation solution and the reserved supernatant. The unlabeled SBs, tumor lysates, and CpG were replaced by the FITC-labeled SBs, rhodamine-labeled tumor lysates, and DAPIlabeled $\mathrm{CpG}$ to confirm the success of loading. The single or merged fluorescent images were captured by confocal microscopy (FV1000; Olympus, Japan). 


\section{Generation of DCs and detection of DC maturation, activation, and antigen presentation}

Murine bone-marrow-derived dendritic cells (BMDCs) were generated from female C57BL/6 or BALB/c mice 8-10 weeks old as previously reported ${ }^{34}$. The erythrocytes in the isolated bone marrow cells $(1 \times$ $10^{5}$ /well) were depleted, and then cultured in RPMI-1640 media with recombinant murine IL -4 (20 $\mathrm{ng} / \mathrm{ml})$ and GM-CSF $(100 \mathrm{U} / \mathrm{ml})$. The non-adherent cells were gently removed on days 2 and 4 . The adherent cell aggregates were dislodged and removed to another 6 -well plate on day 6 . The cells were cultured for a further 6 days, and the resultant non-adherent cells with typical morphological characteristics of BMDCs were harvested for analysis of DC maturation, activation, and crosspresentation. Briefly, BMDCs $\left(1 \times 10^{6}\right.$ cells $\left./ \mathrm{ml}\right)$ were incubated with an OVA-B16 formulation of LC, SB-C, SB-L, or SB-LC for $24 \mathrm{~h}$ in 12-well plates. Thereafter, the BMDCs were collected and stained with antibodies for 30 min at $4^{\circ} \mathrm{C}$ to examine the presence of markers related to DC maturation (CD86, CD80, and CD40) and antigen presentation (SIINFEKL-MHC I and MHC II) by flow cytometry (CyFlow Cube 6; Sysmex, Japan). The antibodies used in this experiment were as follows: APC-Cy7-CD45, PE-CD86, FITCCD80, PE-CD40, APC-SIINFEKL-MHC I, and PerCP-eFluor 450-MHC II (all BioLegend; CA, USA) in a 1:250500 solution. To determine the state of DC activation, the titre of TNF- $a$, IFN- $\gamma$, or IL- 6 secreted by BMDCs in the supernatant was detected by flow cytometry (CyFlow Cube 6) using a CBA Mouse Inflammation Kit (BD) following the manufacturer's protocols. The data generated from the test samples were quantified and compared to a standard curve that was generated from standard samples using FCS EXPRESS V5 software (De Novo Software, Pasadena, CA).

\section{Detection of SB uptake and its influence on antigen cross- presentation by DCs}

Uptake of SB and its influence on antigen cross-presentation by DCs were determined by flow cytometry (CyFlow Cube 6) in the presence or absence of blocking antibodies against PAR1 and MR (BioLegend) in a 1:250-500 solution, and were directly visualized by confocal microscopy (FV1000, Olympus). To evaluate PAR1- or MR-mediated uptake, BMDCs were incubated for $1 \mathrm{~h}$ with a sufficient concentration of anti-PAR1 and anti-MR antibodies alone or combination to completely block the receptors. Following two washes with PBS, the BMDCs were incubated with comparable FITC-labeled SBs or SEs, and the mean fluorescence intensity (MFI) of the BMDCs, indicating the level of SB or SE uptake, was detected by flow cytometry (CyFlow Cube 6); the Cyt D influence on the uptake of SB or SE was also detected in this manner. To understand whether different vaccine formulations (LC, SB-C, SB-L, and SB-LC) were internalized by BMDCs, the FITC-labeled SBs or SEs were replaced by different formulations of tumor lysates (L) labeled with FITC or CpG (C) with DAPI. To investigate the trafficking of SB in BMDCs, the BMDCs were incubated with different formulations of SB (SB-C, SB-L, and SB-LC) labeled the ingredient tumor lysates (L) with FITC for $5 \mathrm{~h}$. Thereafter, the early endosomes were labeled with polyclonal rabbit anti-murine early endosome antigen-1 (EEA1, Abcam) in a 1:250-500 solution followed by Texas Red 
anti-rabbit IgG (KPL) in a 1:250-500 solution, and the lysosomes were stained with LysoTracker Red (Invitrogen) in a 1:250-500 solution. The resultant fluorescent images were captured by confocal microscopy (FV1000, Olympus). To investigate the pathways involved in cross-presentation, BMDCs were incubated with different vaccine formulations in the presence or absence of the proteasome inhibitor MG132 or the lysosome protease inhibitor leupeptin, and the expression of SIINFEKL-MHC I was detected by flow cytometry (CyFlow Cube 6).

\section{Establishment of tumor models and vaccination strategies}

In this study, OVA-B6 (B16), CT26, and 4T1-luciferase cell lines (ATCC, Manassas, VA) were used to establish tumor models. The use of mice in this study was approved by the Animal Use and Care Committee of Hainan Medical University. To establish therapeutic tumor models, $5 \times 10^{6}$ tumor cells were subcutaneously injected into the right flank of syngeneic female mice aged 6-8 weeks. Thereafter, the tumor-bearing mice were randomly divided into four groups ( $n=10$ mice per group) and subcutaneously injected with different vaccine formulations (LC, SB-C, SB-L, or SB-LC) containing comparable does of corresponding tumor lysates $(10 \mu \mathrm{g})$ or $\mathrm{CpG}(3 \mu \mathrm{g})$ in $100 \mu$ PBS on day 1 and day 8 after tumor cell inoculation as previously described ${ }^{35}$. In contrast to establish prophylactic models, the mice in this study were first subcutaneously injected with different vaccine formulations on day -7 and 0 , and the tumor cells were injected on day 1 . The OVA-B16 and CT26 models were established in C57BL/6 and BALB/C syngeneic mice, respectively. The tumor images and volumes at different time points were obtained by a portable measuring device (TM900; Peira, Belgium). In addition, 4T1-luciferase cells were used to establish metastatic model in syngeneic BALB/c mice using a similar protocol as a previously established prophylactic model by tail vein injection of $5 \times 10^{6}$ cells. To judge the anti-metastatic effects of the vaccinations, the mice were intraperitoneally injected with ${ }_{D}$-fluorescein potassium $(300 \mathrm{mg} / \mathrm{kg})$, and the luminescence intensity images were captured by an IVIS lumina II imaging system (Caliper Life Sciences).

\section{Observations of adverse side effects}

To observation the potential side effects induced by the vaccination, normal mice aged 6-8 weeks were vaccinated only with various formulations as in the protocol of prophylactic models, but not establishment of tumor models, and the following parameters were observed as potential side effects for 180 days. The body weight and life span of the mice were recorded every month, and other gross measures, including ruffled fur, feeding, and behavior, were also observed. Fertility was also observed as a parameter of potential toxicity, as reported previously. In brief, 2 weeks after the second vaccination, female mice were placed in cages with males, and both the number of days until parturition and the number of pups were recorded. At the end of the observation period, tissues of major organs, including the heart, liver, spleen, lung, and kidney, were collected and fixed in 10\% neutral-buffered formalin and embedded in paraffin. Thereafter, $3-5 \mu \mathrm{m}$ sections were stained with haematoxylin and eosin (H\&E), and 
the tissue structures and cellular morphology were observed under microscopy (). The sections of major organs were also stained with FITC-conjugated anti-mouse $\lg$ G, IgM, or $\lg \mathrm{A}$ and observed under fluorescence microscopy to determine whether autoantibody deposition was present. Furthermore, blood samples were extracted by tail vein 1 day after terminating treatment. The white blood cells (RBCs), red blood cells (RBCs), and platelets were counted as a measure of bone marrow toxicity, and the levels of creatinine, aspartate transaminase (AST), alanine transaminase (ALT), and lactic dehydrogenase (LDH) were recorded as measures of heart, liver, kidney, or other pan-organ toxicity using a small animal biochemical analyser (AAA, BB, USA).

\section{Detection of cellular immunity}

To test the efficiency of antigen-specific CTLs, splenocytes from the mice vaccinated with OVA-B16 formulations were double stained with SIINFEKL/H-2 $\mathrm{K}^{\mathrm{b}}$ peptide-MHC tetramers (Becton Dickinson) and PE-Cy7-anti-CD8a antibody (BioLegend) in a 1:250-500 solution, and then analysed by flow cytometry (CyFlow Cube 6). CTL-induced direct tumor lysis by splenocytes (effector) against B16 and CT 26 tumor cells (target) was detected using a CytoTox 96 cytotoxicity assay kit (Promega, Madison, WI) according to the manufacturer's instructions and as previously reported ${ }^{33}$. In brief, splenocytes and tumor cells at various ratios (5-40:1) were plated on 96-well plates in a final volume of $100 \mu \mathrm{l}$ and then incubated in a humidified atmosphere $\left(5 \% \mathrm{CO}_{2}\right.$ at $\left.37^{\circ} \mathrm{C}\right)$ for $6 \mathrm{~h}$. The aliquots and reconstituted substrates (each $\left.50 \mu \mathrm{l}\right)$ were transferred to another flat-bottom 96-well plate, and incubated in the dark at room temperature for $50 \mathrm{~min}$. The experiment was terminated by adding $50 \mu \mathrm{l}$ stop solution, and the optical density (OD) values at $492 \mathrm{~nm}$ were obtained using an ELX808IU microplate reader (Bio-Tek, USA). The percentage of target cell death at each E:T ratio was calculated as reported previously ${ }^{36}$.

In this study, we adapted an in vivo method from a previous study to detect the specific CTL response. Briefly, $\mathrm{H} 22$ cells were stained with CFSE $(7 \mathrm{mM})$ for $20 \mathrm{~min}$, and $5 \times 10^{6}$ cells were injected into the abdominal cavity of BALB/c mice vaccinated with various vaccine formulations. After $24 \mathrm{~h}$, the mice were sacrificed, and the proliferated $\mathrm{H} 22$ and immune cells in the abdominal cavity were collected. These cells were further stained with Cy5-anti-CD 45 antibody (Abcam), and the $\mathrm{CD} 45^{-} \mathrm{H} 22$ cells were gated from the CD $45^{+}$immune cells to analyze cell proliferation. In addition, the total cell number in each group was used to indirectly calculate the percentage of $\mathrm{H} 22$ cell lysis using the following formula: Percentage of specific lysis $=(1-$ vaccinated group/unvaccinated group $) \times 100$.

The splenocytes secreting IFN- $y$, TNF- $a$, and IL-12 were isolated from the spleens of mice vaccinated with the various vaccine formulations ${ }^{33}$. These splenocytes $\left(1 \times 10^{6}\right)$ were stained with the corresponding monoclonal antibodies conjugated with FITC-anti-IFN-y, PE-anti-TNF-a, or Percp-Cy5.5-antiIL12 antibody (BD, NJ, USA) for 30 min at $4^{\circ} \mathrm{C}$. The stained cells were detected by flow cytometry (CyFlow Cube 6 and FACS Canto II), and the data were analysed with FlowJo Software version 10 (Tree Star, Ashland, OR). 


\section{Analysis of humoral immunity}

$\mathrm{IgG}$ in the serum of mice vaccinated with various vaccine formulations in both B16 and CT26 models was detected by western blotting ${ }^{36}$. The proteins of the B16 or CT26 cell lysates were separated in $12 \%$ sodium dodecyl sulfate polyacrylamide gel electrophoresis (SDS-PAGE) gels and then transferred onto polyvinylidene difluoride membrane (Bio-Rad) using a Mini Transblot device (Bio-Rad, Hercules, CA).

Thereafter, the membranes were stained with anti-mouse IgG antibodies (Abcam) at a 1:150 dilution after blocking by $10 \%$ non-fat milk at $4^{\circ} \mathrm{C}$ for $2 \mathrm{~h}$. Finally, the membranes were incubated with chemiluminescence substrates $A$ and $B(1: 1)$ for 30 min, and images were captured using an ECL system (Amersham Biosciences, UK).

The numbers of splenic monocytes secreting tumor-specific antibodies were detected by ELISPOT assay. In brief, B16 or CT26 tumor lysate ( $5 \mu \mathrm{g} /$ well) was first fixed onto the polyvinylidene difluoride membrane fixed to the bottom of ELISPOT Plates (Millipore, Billerica, MA). The splenic monocytes from the mice vaccinated with various vaccine formulations were incubated in the plates for $6 \mathrm{~h}$, in which the IgG antibody secreted by the splenocytes served as the primary antibody. Thereafter, the secondary antimouse IgG antibody was used to stain the ELISPOT membrane following a procedure similar to the aforementioned Western blotting assay, but the results appeared as spots and not bands. In addition, the titres of IgG and its subtypes were detected by ELISA kits (Wuhan Boster Biological Technology, China), according to the manufacturer's protocol ${ }^{36}$.

\section{Adoptive transfer of splenocytes and antibodies}

Adoptive transfer of splenocytes from mice vaccinated with different vaccine formulations to newly B16bearing and CT26-bearing mice was performed as reported previously ${ }^{36}$. In brief, the splenocytes from the mice vaccinated with the different formulations were isolated on day 14 after vaccination. The B16 or CT26 tumor models were established in new recipient mice $(n=5)$ by injection of corresponding tumor cells in the same manner as the previously mentioned therapeutic protocol. Subsequently, $5 \times 10^{6}$ splenocytes per mouse were transferred into the recipient mice by tail vein on day 0 after the tumor models were established. The transfers were repeated two more times on days 5 and 10 . On day 18 , the tumor images and volumes were captured using a portable TM900 system (Peira, Belgium). To adoptively transfer antibodies, the pooled sera from mice vaccinated with different formulations were passed through a chromatography column (CM Affi-gel Blue Gel Kit; Bio-Rad) to acquire the purified immunoglobulins. These antibodies (50 mg/ $\mathrm{kg}$ in each mouse) were injected into tumor-bearing recipient mice three times at the same time points as the mice that received the splenocytes. The tumor images and volumes were then captured by a TM900 portable measuring device.

\section{Calculation of synergistic indexes}


In this study, we investigated whether the cellular and antibody immunities synergized to combat tumor growth. Splenocytes and antibodies isolated from H22 SB-LC-vaccinated mice using the same protocols as adoptive transfer were used in this study. Twenty BALB/c mice were injected with CFSE-labeled $\mathrm{H} 22$ cells and then randomly divided into four groups. The mice were then intraperitoneally injected with PBS, splenocytes $\left(5 \times 10^{6}\right)$, and antibodies $(50 \mathrm{mg} / \mathrm{kg}$ ) either alone or in combination. The cells in the abdominal cavity were collected, stained, and gated by flow cytometry (CyFlow Cube 6) as outlined previously. The synergistic indexes (SI) were calculated from the number of $\mathrm{CD} 45^{-} \mathrm{H} 22$ cells ${ }^{23}$. In brief, the observed relative ratio (ORR) of the number of cells in the splenocyte, antibody, or combination group was calculated by dividing the number of cells in each group by that in the PBS group. The expected relative ratio (ERR) of the combination group was calculated by multiplying the ORR of the splenocyte group with that of the antibody group; then, the $\mathrm{SI}$ of the combination was calculated as $\mathrm{SI}=\mathrm{ERR} / \mathrm{ORR}$, where $\mathrm{SI}>1$ indicates a synergistic effect.

\section{Depletion of $\mathrm{CD4}^{+}$or $\mathrm{CD8}^{+}$lymphocytes}

Depletion of $\mathrm{CD}^{+}$or $\mathrm{CD} 8^{+}$lymphocytes was performed as reported previously ${ }^{36}$. In brief, rat monoclonal anti-CD4 IgG (clone GK 1.5) or an anti-CD8 IgG (clone 2.43) antibody isolated from the corresponding hybridoma (ATCC, Manassas, VA, USA) was intraperitoneally injected into B16- or CT26-bearing mice (1 $\mathrm{mg} /$ mouse) four times in 4-day intervals, with the first injection on day 0 . The splenocytes were analysed by flow cytometry (CyFlow Cube 6) to ensure that the efficacy of cell depletion was greater than $98 \%$. Thereafter, the depleted mice were used to establish a B16 or CT26 model with subcutaneous injection of $5 \times 10^{5}$ tumor cells. The tumor images and volumes were captured by a TM900 portable measuring device on day 18 after tumor cell injection as mentioned previously. Meanwhile, the titres of IgG antibodies specific to B16 or CT26 cells in the sera were collected and detected by ELISA 18 days after tumor cell injection as outlined above.

\section{Evaluation of the immunosuppressive microenvironment}

Tumor-infiltrating lymphocytes (TILs), including $C D 8^{+} \mathrm{T}$ cells, MDSC, and Treg, were first observed in tumor sections. Tumour tissues from the mice vaccinated with the different formulations were removed 18 days after tumor cell injection, and the frozen sections $(3-5 \mu \mathrm{m})$ were stained with the following fluorescence-labeled antibodies: APC-Cy7-anti-CD8, PE-anti-CD11b, FITC-anti-Gr-1, and PerCP-anti-Foxp3 (BioLegend) at 1:250-500 dilutions. The fluorescent images were captured and merged by confocal microscopy (FV1000, Olympus). In addition, TILs were generated from different tumor masses and analysed by flow cytometry (CyFlow Cube 6). In brief, tumor masses from mice vaccinated with various formulations were removed by forceps and cut into pieces approximately $1 \mathrm{~mm}^{3}$ in size, and subsequently treated with DMEM media containing collagenase $(1 \mathrm{mg} / \mathrm{ml})$ and DNase I (Sigma) for $3 \mathrm{~h}$ at $37^{\circ} \mathrm{C}$. The resultant cell suspension was treated with Lysing Buffer (BD Pharm) and passed through a 70-mm cell strainer. Thereafter, the cells were re-suspended in $5 \mathrm{ml}$ Percoll (33\%) and centrifuged at 3,000 
rpm for $15 \mathrm{~min}$. In addition to the abovementioned fluorescent antibodies, other antibodies, including FITC-anti-CD45, APC-anti-CD4, APC-Cy7-anti-CD25, PE-anti-CD206, and FITC-anti-F4/80, were used. The $\mathrm{CD}_{4} 5^{+}$single or combined with $\mathrm{CD} 4^{+}$or $\mathrm{CD} 11 \mathrm{~b}^{+}$double-positive TILs were gated out from the mixture of CD45 $5^{+}$TILs and CD45 tumor cells; these cell phenotypes were further analysed by flow cytometry to identify IFN- $\gamma$-secreting CD $8^{+}$T cells, MDSC, Treg, and M2-type ATMs.

\section{Detection of tumor cell ferroptosis}

Lipid reactive oxygen species (ROS) were assessed by BODIPY-C11 staining (Thermo Fisher) in B16- or CT26-bearing mice or in B16 or CT26 cells in vivo as previously reported ${ }^{30}$. To quantify the relative ROS in tumor tissues from mice vaccinated with SB-C or SB-LC treated with or without $10 \mathrm{mg} / \mathrm{kg}$ ferroptosisinhibitor liproxstatin-1 (Cayman), the images and tumor volumes were monitored using a TM900 portable measuring device, and the mice were sacrificed to collect the tumor tissues on day 15 after tumor cell injection. The tumor tissues were resected and cut into small pieces, which were then mechanically minced in a cell strainer $(100 \mu \mathrm{M})$ and washed twice with PBS. The mixture of the tumor and TILs was enriched by Ficoll (Sigma-Aldrich) centrifugation. The cell pellet was stained with anti-CD45 antibody (BioLegend) followed by BODIPY 581/591 C11 (Thermo Fisher) at 1:250-500 dilutions and analysed by flow cytometry (CyFlow Cube 6) after straining through a $40-\mu \mathrm{M}$ cell strainer. The CD45 tumor cells were distinguished from $\mathrm{CD} 45^{+}$TILs or splenocytes by the flow cytometry gating strategy. For BODIPY 581/591 C11 staining, the fluorescence intensity from both oxidized C11 (FITC signal) and non-oxidized C11 (PE signal) was captured. The MFI ratio of the FITC signal to the PE signal was calculated for each sample. The data were normalized to the SB-C samples as shown by the relative lipid ROS.

To detect the relative ROS in vivo, B16 or CT26 cells (10,000 cells) were seeded with the same amount of splenocytes from the mice vaccinated with SB-C or SB-LC in the presence or absence of ferrostatin-1 (10 $\mu \mathrm{M})$ or the ferroptosis inducer RSL3 (1 $\mu \mathrm{M}$, Cayman) in 24-well plates for $20 \mathrm{~h}$. Subsequently, the cells were trypsinized and collected for staining and flow cytometric analysis using the abovementioned method to analysed the relationship between the CD45 tumor cells and CD $45^{+} \mathrm{TILs}$. These cells were also used to analyse ferroptosis-related cell death. In brief, after $20 \mathrm{~h}$ co-culture, the cells were collected and re-suspended in PBS containing $1 \mu \mathrm{g} / \mathrm{ml}$ 7-aminoactinomycin D (7-AAD) for $10 \mathrm{~min}$. The CD45-tumor cells were gated from the $C D 45^{+}$splenocytes, and the dead cells were calculated as the proportion of 7$\mathrm{ADD}^{+}$cells by flow cytometric analysis.

To investigate the relationship between ferroptosis and immunity in vivo, SB-C- or SB-LC-vaccinated B16or CT26-bearing mice were treated with liproxstatin-1, and tumor tissues were collected. The TIL phenotypes related to IFN-y-secreting CD ${ }^{+} \mathrm{T}$ cells, MDSC, Treg, and M2-type ATMs were analysed by flow cytometry as outlined above. 


\section{Statistical Analysis}

The data in this study were analyzed by GraphPad-Prism Software version 9.0.0 for Windows (San Diego, California USA), and presented as the mean \pm standard deviation (SD). As all the data concern more than two groups, one- or two-way analysis of variance (ANOVA) followed by a Tukey's honest significant difference test was performed to compare the differences between groups. Animal survival was graphed as Kaplan-Meier survival curves and analysed with the log-rank (Mantel-Cox) test. ${ }^{*}<0.05, * \star<0.01, * \star \star$ $<0.001, \star \star \star \star<0.0001$.

\section{Declarations}

The authors declare no competing interests.

\section{References}

1.Hsieh, M. H. \& Mentink-Kane, M. M. Smallpox and Dracunculiasis: The Scientific Value of Infectious Diseases That Have Been Eradicated or Targeted for Eradication. Is Schistosomiasis Next? PLoS pathogens 12,e1005298 (2016).

2.Taylor, P. R., Gordon, S. \& Martinez-Pomares, L. The mannose receptor: linking homeostasis and immunity through sugar recognition. Trends in immunology 26,104-110 (2005).

3.Tang, D., Kang, R., Coyne, C. B., Zeh, H. J. \& Lotze, M. T. PAMPs and DAMPs: signal Os that spur autophagy and immunity. Immunological reviews 249,158-175 (2012).

4.Bianchi, M. E. DAMPs, PAMPs and alarmins: all we need to know about danger. Journal of leukocyte biology 81,1-5 (2007).

5.Demento, S. L., Siefert, A. L., Bandyopadhyay, A., Sharp, F. A. \& Fahmy, T. M. Pathogen-associated molecular patterns on biomaterials: a paradigm for engineering new vaccines. Trends in biotechnology 29,294-306 (2011).

6.Doshi, N. \& Mitragotri, S. Macrophages recognize size and shape of their targets. PloS one 5,e10051 (2010).

7.Gratton, S. E. et al. The effect of particle design on cellular internalization pathways. Proceedings of the National Academy of Sciences of the United States of America 105,11613-11618 (2008).

8.Ni, D. et al. Biomimetically Engineered Demi-Bacteria Potentiate Vaccination against Cancer. Advanced science 4,1700083 (2017).

9.Bevan, M. J. Cross-priming. Nature immunology 7,363-365 (2006). 
10.Joffre, O. P., Segura, E., Savina, A. \& Amigorena, S. Cross-presentation by dendritic cells. Nature reviews. Immunology 12,557-569 (2012).

11.Limmathurotsakul, D. et al. Predicted global distribution of Burkholderia pseudomallei and burden of melioidosis. Nature microbiology 1,15008 (2016).

12.Wiersinga, W. J. et al. Melioidosis. Nature reviews. Disease primers 4,17107 (2018).

13.Gassiep, I., Armstrong, M. \& Norton, R. Human Melioidosis. Clinical microbiology reviews 33 (2020).

14.Kim, J. H. et al. Phase I/II study of immunotherapy using autologous tumor lysate-pulsed dendritic cells in patients with metastatic renal cell carcinoma. Clinical immunology 125,257-267 (2007).

15. Holtl, L. et al. Immunotherapy of metastatic renal cell carcinoma with tumor lysate-pulsed autologous dendritic cells. Clinical cancer research: an official journal of the American Association for Cancer Research 8,3369-3376 (2002).

16.Kandalaft, L. E. et al. Autologous lysate-pulsed dendritic cell vaccination followed by adoptive transfer of vaccine-primed ex vivo co-stimulated T cells in recurrent ovarian cancer. Oncoimmunology 2,e22664 (2013).

17.Ringel, A. E. et al. Obesity Shapes Metabolism in the Tumor Microenvironment to Suppress Anti-Tumor Immunity. Cell 183,1848-1866 e1826 (2020).

18.Burgdorf, S., Kautz, A., Bohnert, V., Knolle, P. A. \& Kurts, C. Distinct pathways of antigen uptake and intracellular routing in CD4 and CD8 T cell activation. Science 316,612-616 (2007).

19.Burgdorf, S. \& Kurts, C. Endocytosis mechanisms and the cell biology of antigen presentation. Current opinion in immunology 20,89-95 (2008).

20.Shen, K. Y. et al. Molecular mechanisms of TLR2-mediated antigen cross-presentation in dendritic cells. Journal of immunology 192,4233-4241 (2014).

21.Shen, L. \& Rock, K. L. Priming of T cells by exogenous antigen cross-presented on MHC class I molecules. Current opinion in immunology 18,85-91 (2006).

22.Fehres, C. M., Unger, W. W., Garcia-Vallejo, J. J. \& van Kooyk, Y. Understanding the biology of antigen cross-presentation for the design of vaccines against cancer. Frontiers in immunology 5,149 (2014).

23.Tan, G. H. et al. Combination of low-dose cisplatin and recombinant xenogeneic endoglin as a vaccine induces synergistic antitumor activities. International journal of cancer 112,701-706 (2004).

24.Schupp, J. et al. Targeting myeloid cells in the tumor sustaining microenvironment. Cellular immunology 343,103713 (2019). 
25.Bottcher, J. P. et al. NK Cells Stimulate Recruitment of cDC1 into the Tumor Microenvironment Promoting Cancer Immune Control. Cell 172,1022-1037 e1014 (2018).

26.McGranahan, N. \& Swanton, C. Cancer Evolution Constrained by the Immune Microenvironment. Cell 170,825-827 (2017).

27.Yang, W. S. et al. Regulation of ferroptotic cancer cell death by GPX4. Cell 156,317-331 (2014).

28.Conrad, M., Angeli, J. P., Vandenabeele, P. \& Stockwell, B. R. Regulated necrosis: disease relevance and therapeutic opportunities. Nature reviews. Drug discovery 15,348-366 (2016).

29.Linkermann, A. et al. Synchronized renal tubular cell death involves ferroptosis. Proceedings of the National Academy of Sciences of the United States of America 111,16836-16841 (2014).

30.Wang, W. et al. CD8(+) T cells regulate tumour ferroptosis during cancer immunotherapy. Nature 569,270-274 (2019).

31.Elliott, M. R. \& Ravichandran, K. S. The Dynamics of Apoptotic Cell Clearance. Developmental cell 38,147-160 (2016).

32.Fang, Y. et al. First genome sequence of a Burkholderia pseudomallei Isolate in China, strain BPC006, obtained from a melioidosis patient in Hainan. Journal of bacteriology 194,6604-6605 (2012).

33.Huang, F. Y. et al. Microencapsulation of tumor lysates and live cell engineering with MIP-3 alpha as an effective vaccine. Biomaterials 53,554-565 (2015).

34.Huang, F. Y. et al. Induction of enhanced immunogenic cell death through ultrasound-controlled release of doxorubicin by liposome-microbubble complexes. Oncoimmunology 7,e1446720 (2018).

35.Tan, G. H. et al. Active immunotherapy of tumors with a recombinant xenogeneic endoglin as a model antigen. European journal of immunology 34,2012-2021 (2004).

36. Huang, F.-Y. et al. A recombinant oncolytic Newcastle virus expressing MIP-3a promotes systemic antitumor immunity. Journal for immunotherapy of cancer 8,e000330 (2020).

\section{Figures}



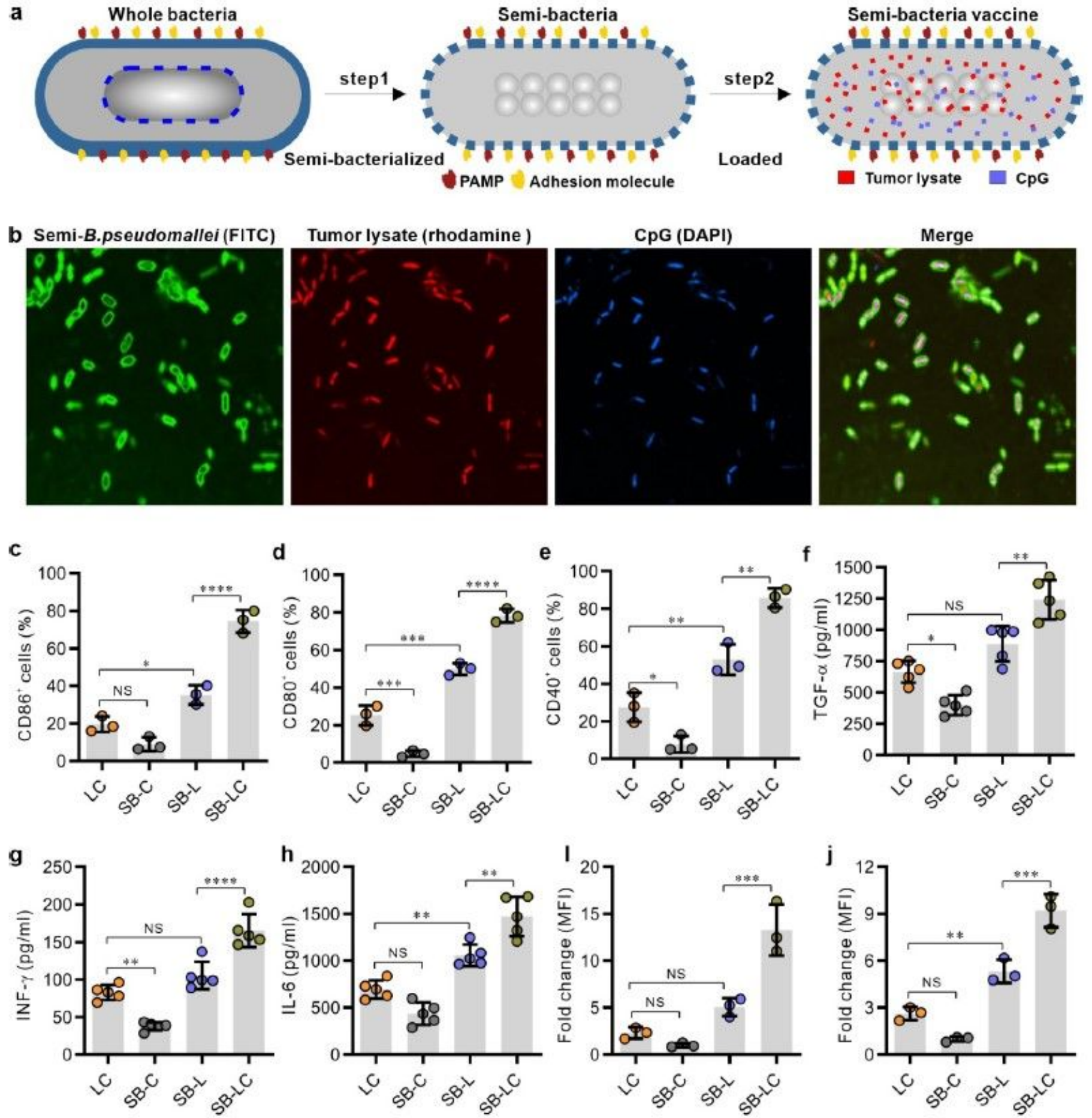

\section{Figure 1}

Engineered Porous/Hollow Semi-Burkholderia Pseudomallei (SB) Loading Tumor Lysates and CpG Promotes Dendritic Cell (DC) Maturation and Antigen Cross-Presentation (A) Schematic illustration of the fabrication of SB loading tumour lysates and CpG. (B) Confocal microscopy images showing colocalization of SB (FITC), tumour lysates (rhodamine), and CpG (DAPI). Scale bar: $2 \mu \mathrm{m}$. (C-E) The percentage of $\mathrm{CD} 86+(\mathrm{C}), \mathrm{CD} 80+(\mathrm{D})$, and $\mathrm{CD} 40+\mathrm{BMDCs}$ co-cultured with the indicated formulations (mean $\pm S D, n=3)$. $(F-H)$ The titres of TGF- $a(F)$, INF-Y $(G)$, and IL-6 $(H)$ in the supernatants co-cultured with BMDCs and the indicated formulations (mean $\pm S D, n=5$ ). (I and $J$ ) The MFI fold change of the 
SIINFEKL-MHC I (I) and MHC II (J) on the BMDCs co-cultured with the indicated formulations (mean \pm SD, $\mathrm{n}=3$ ). One-way ANOVA with Tukey multiple comparisons: $*<0.05, * \star<0.01, * \star \star<0.001, * \star \star \star<0.0001$. MFI: Mean fluorescence intensity, BMDCs: Bone-marrow-derived dendritic cells. See also Figure S1.$$
\text { a }
$$
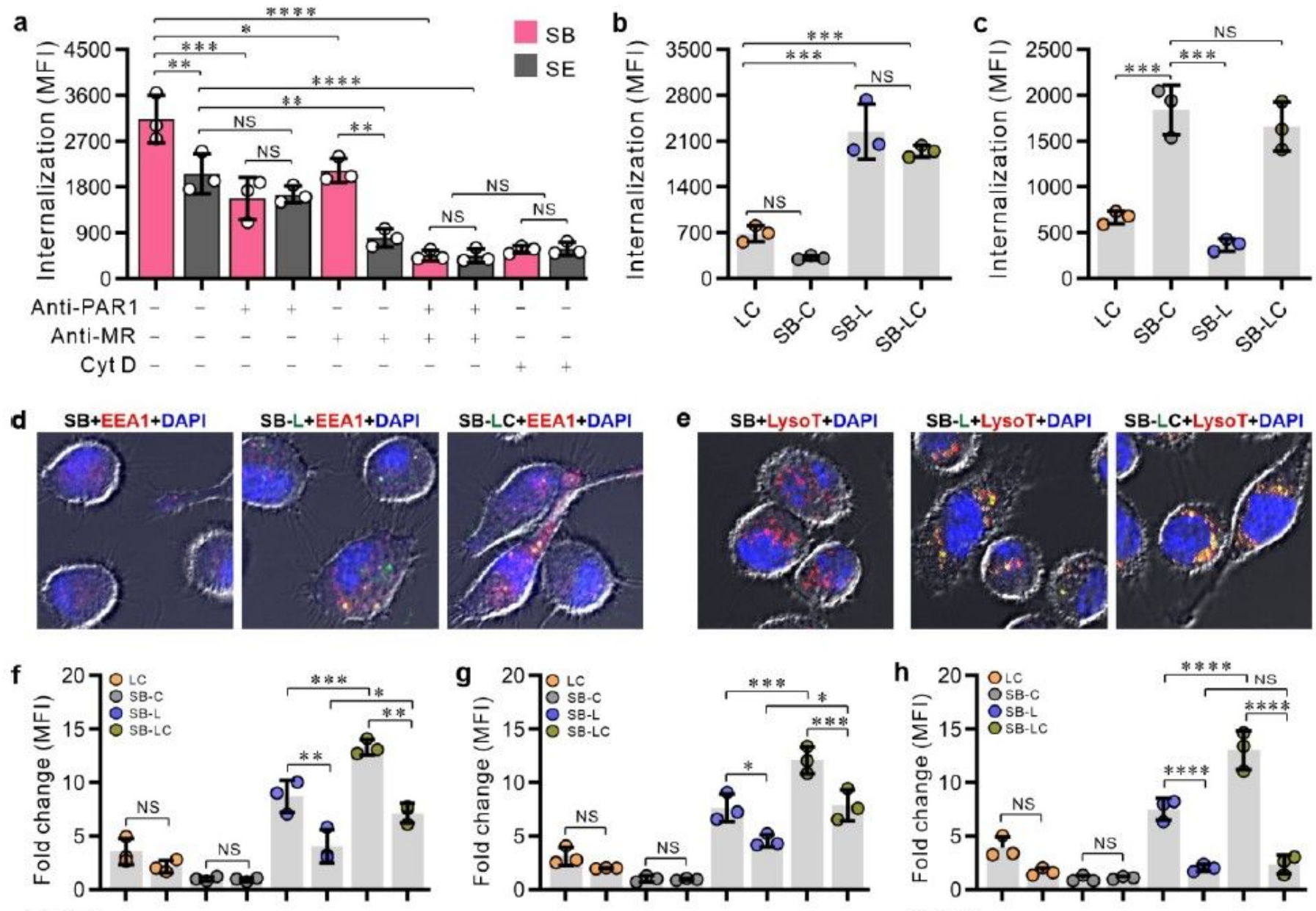

MG132
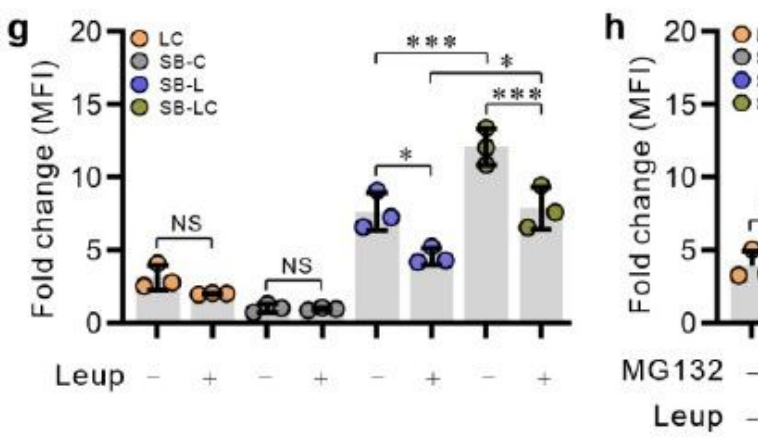

\section{Figure 2}

Adhesion Proteins of B. Pseudomallei Facilitate the Internalization of SB-Loading Antigen and Enhance Cross-Presentation (A) The MFI of the BMDCs co-cultured with FITC-labelled SB or semi-Escherichia coli $(\mathrm{SE})$ in the presence or absence of anti-PAR1, anti-MR, or cytochalasin D (Cyt D) (mean $\pm S D, n=3)$. (B and C) The MFI of the BMDCs co-cultured with the indicated formulations labelled with tumour lysates (B) or $\mathrm{CpG}(\mathrm{C})$ (mean $\pm \mathrm{SD}, \mathrm{n}=3$ ). (D and E) The SB formulations (FITC-labelled tumour lysates; $\mathrm{L}$ ) internalized by BMDCs were conformed to co-localize with EEA1+ endosomes (D) and LysoT(racker Red)labelled lysosomes (E). Scale bar: $50 \mu \mathrm{m}$. (F-H) The MFI fold change of the SIINFEKL-MHC I on the BMDCs co-cultured with the indicated formulations in the presence of MG132 (F), leupeptin (Leup, $G$ ), or in combination with MG132 and Leup $(H)$ (mean $\pm S D, n=3)$. One way ANOVA with Tukey multiple comparisons: $*<0.05, \star \star<0.01, \star \star \star<0.001, \star \star \star \star<0.0001$. MFI: Mean fluorescence intensity, BMDCs: Bone-marrow-derived dendritic cells. 
a
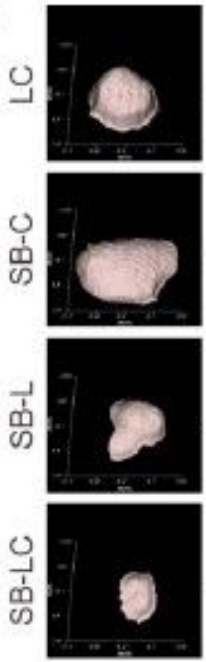
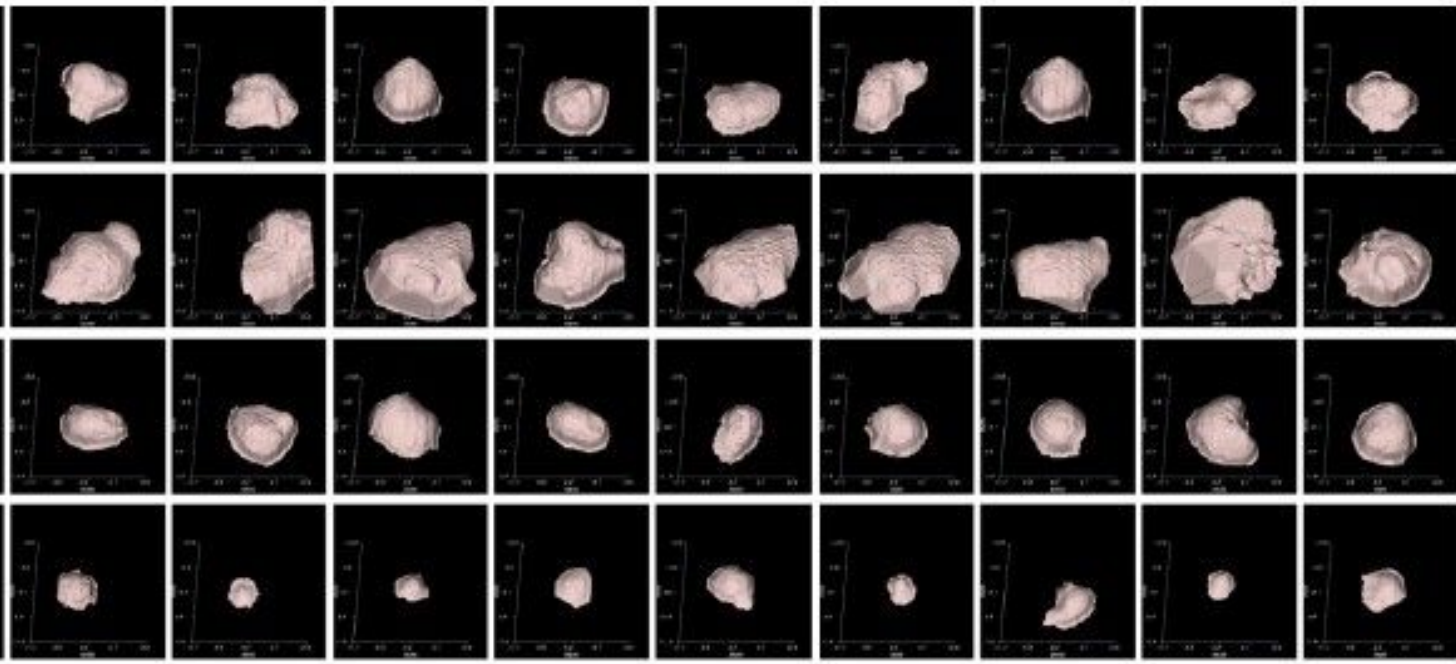

b
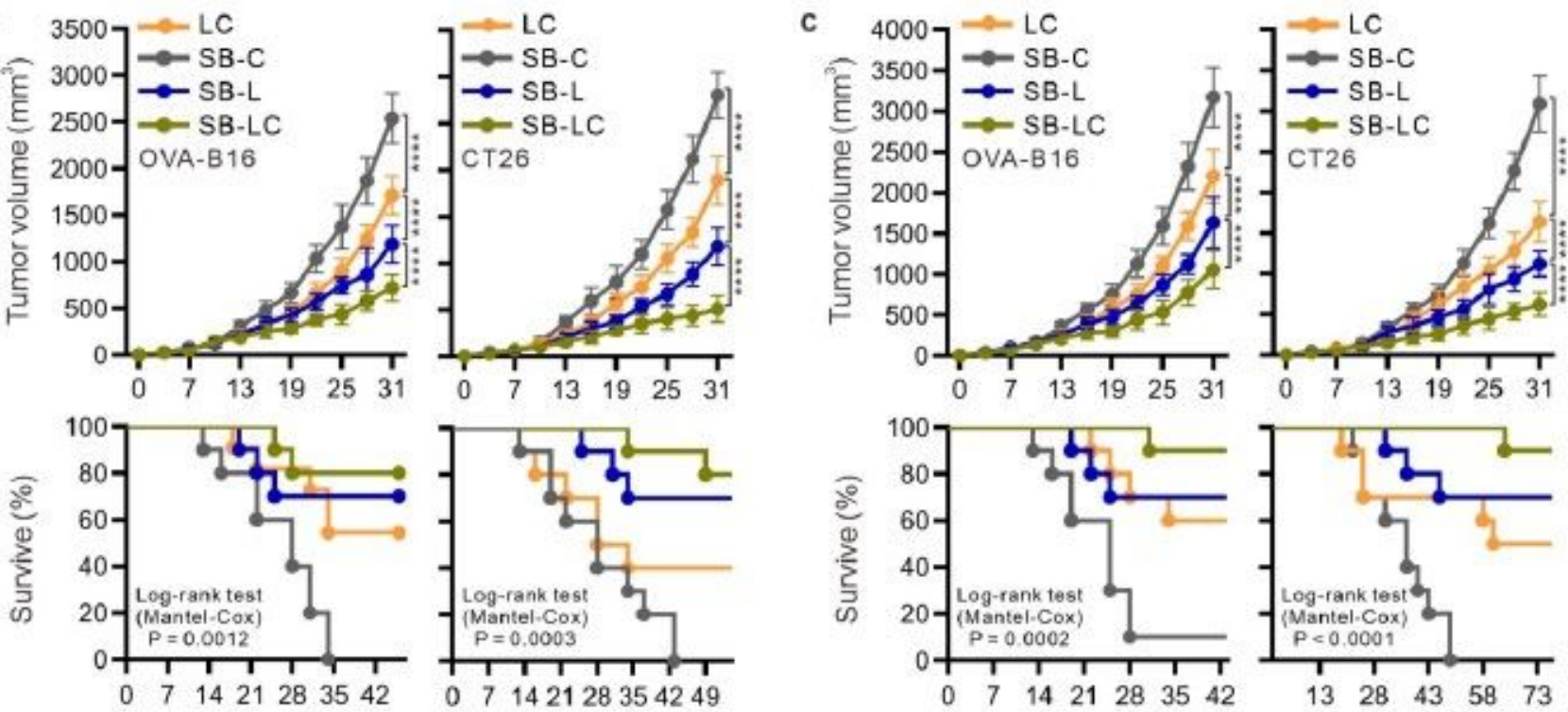

Days after tumor cell innoculation

Days after tumor cell innoculation

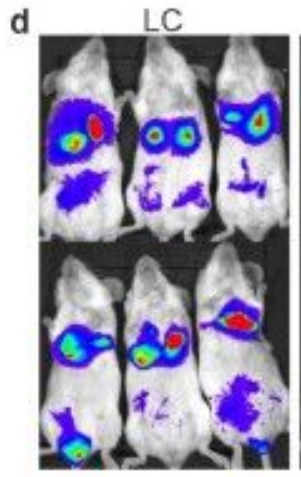

SB-C
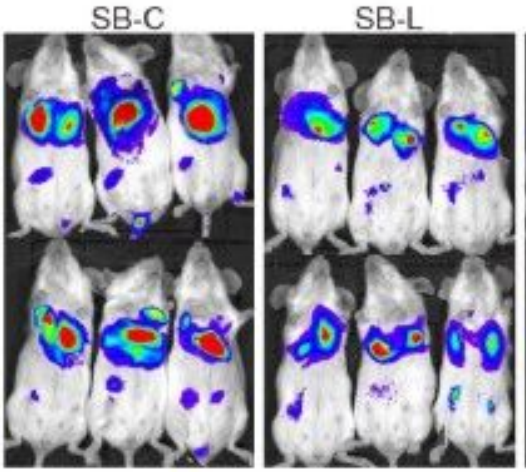

SB-LC
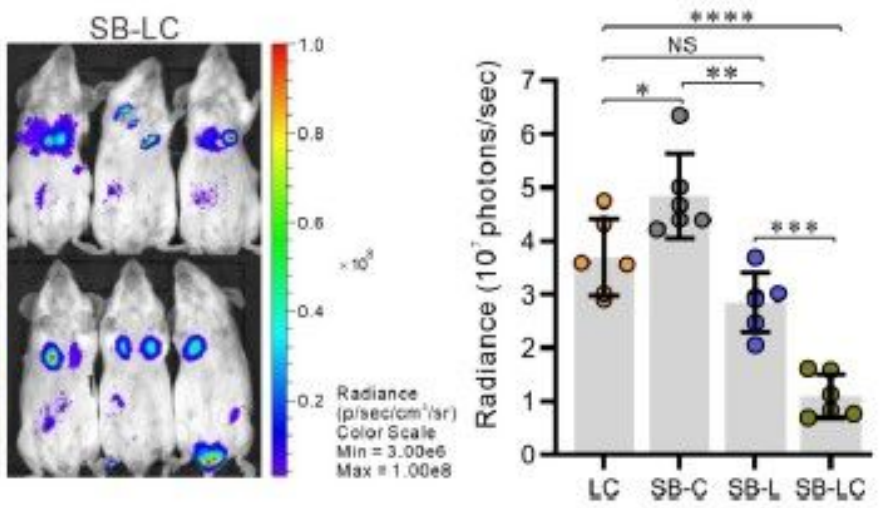

Figure 3

SB-LC Vaccination Induces Therapeutic and Prophylactic Anti-Tumour Effects (A) Images showing the tumour masses of the B16 model on day 18 after tumour cell inoculation $(n=10)$. (B and C) Tumour volume (mean $\pm S D, n=10$ ) and survival rate of both B16 and CT26 mice vaccinated with the indicated formulations using either a therapeutic (B) or prophylactic protocol (C). (D) The luminescence (107 photons/sec) in 4T1 mice vaccinated with the indicated formulations (mean $\pm S D, n=6$ ). Two- (tumor 
volume) or one-way (D) ANOVA with Tukey multiple comparisons, and Log-rank (Mantel-Cox) test

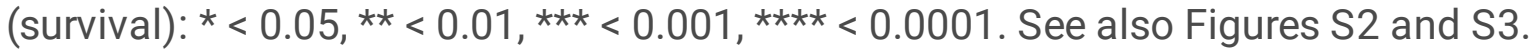
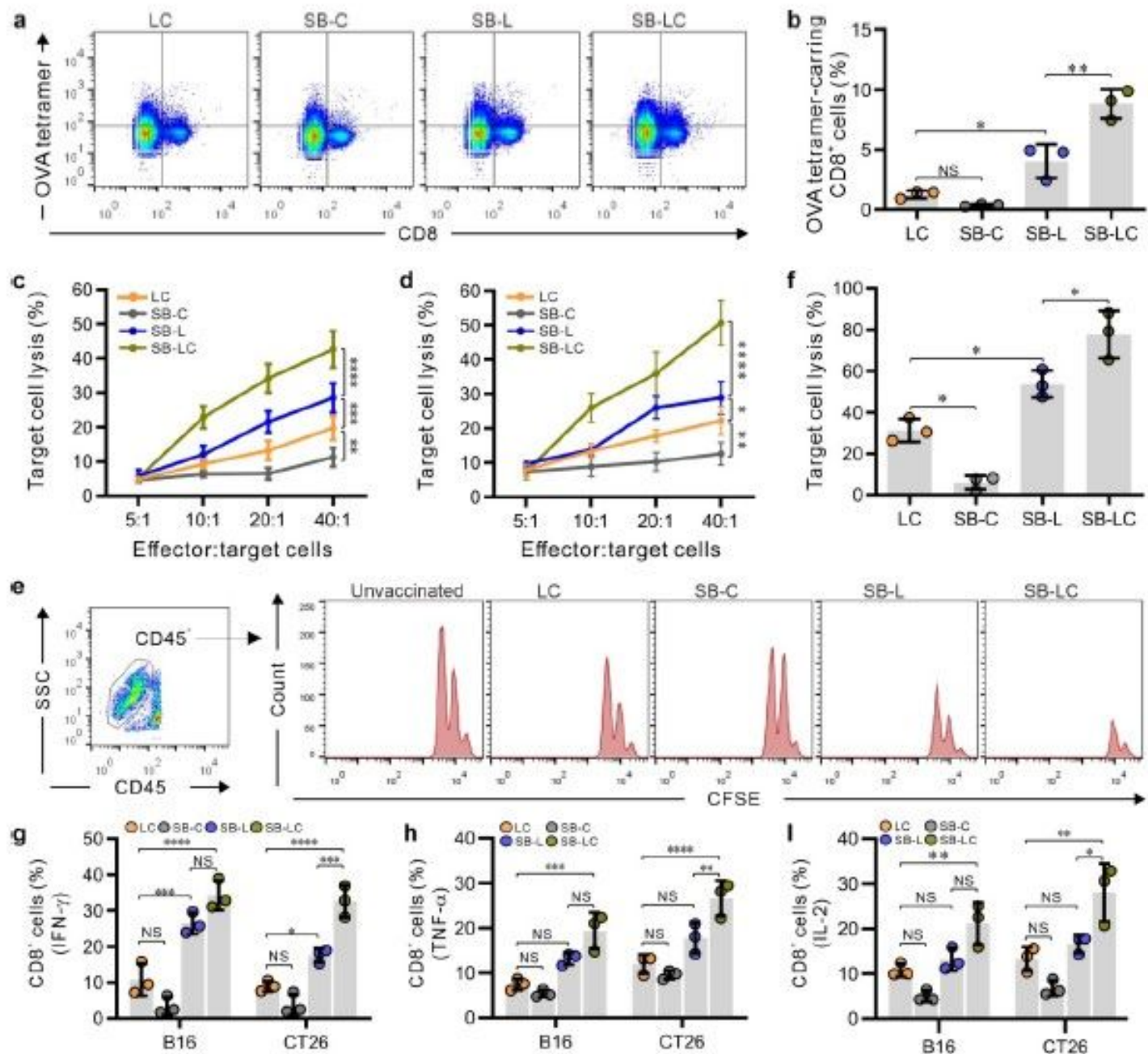

Unvaccinated

Effector:target cells
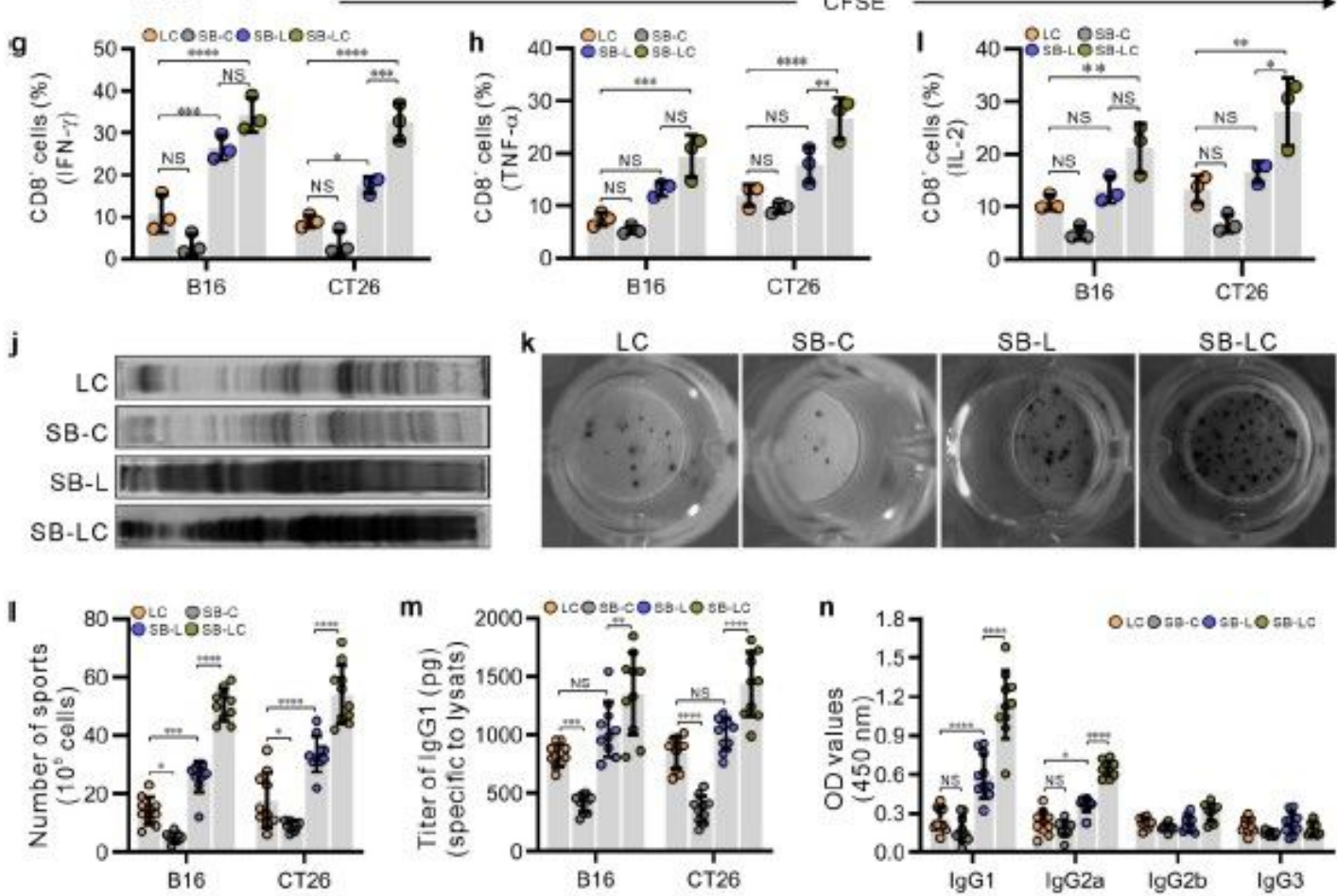

\section{Figure 4}

SB-LC Vaccination Provokes Tumor-Specific Cellular and Humoral Immunity. (A and B) Representative flow cytometry images (A) showing the percentage of the OVA257-264-bound $\mathrm{H}-2 \mathrm{~Kb}$ tetramer-binding CD8+ splenocytes (B) in the OVA-B16-bearing mice vaccinated with the indicated formulations (mean \pm 
SD, $n=3)$. (C and D) CTL response using B16 (C) and CT26 (D) as target cells and the splenocytes from the mice vaccinated with the indicated formulations as effector cells. (E and $F$ ) The in vivo CTL response was examined by injecting the CFSE-labelled $\mathrm{H} 22$ cells into the abdominal cavity to determine the proliferation of $C D 45-$ tumour cells $(E)$ and the percentage of $H 22$ cell lysis $(F)$ (mean $\pm S D, n=3)$. ( $G-I$ ) The percentage of CD8+ splenocytes secreting IFN-y (G), TNF- $a(H)$, and IL-2 (I) from B16- or CT26bearing mice vaccinated with the indicated formulations (mean $\pm S D, n=3)$. (J) Western blot detection of the tumour-specific $\lg G$ in the serum from mice vaccinated with the indicated formulations. $(K$ and $L)$ ELISPOT detection of the splenocytes secreting IgG specific to B16 or CT26 cells $(K)$, and the average number of IgG-secreting cells in 105 splenocytes $(L)$ (mean $\pm S D, n=10)$. ( $M$ and $N$ ) The IgG titre in the serum from B16- or CT26-bearing mice vaccinated with the indicated formulations (M), and the IgG subtypes in the B16-bearing mice $(\mathrm{N})$ (mean $\pm S D, n=10)$. One- (B and F) or two-way $(G-I$ and $L-N)$ ANOVA with Tukey multiple comparisons: $*<0.05, * \star<0.01, * \star \star<0.001, * \star \star \star<0.0001$.
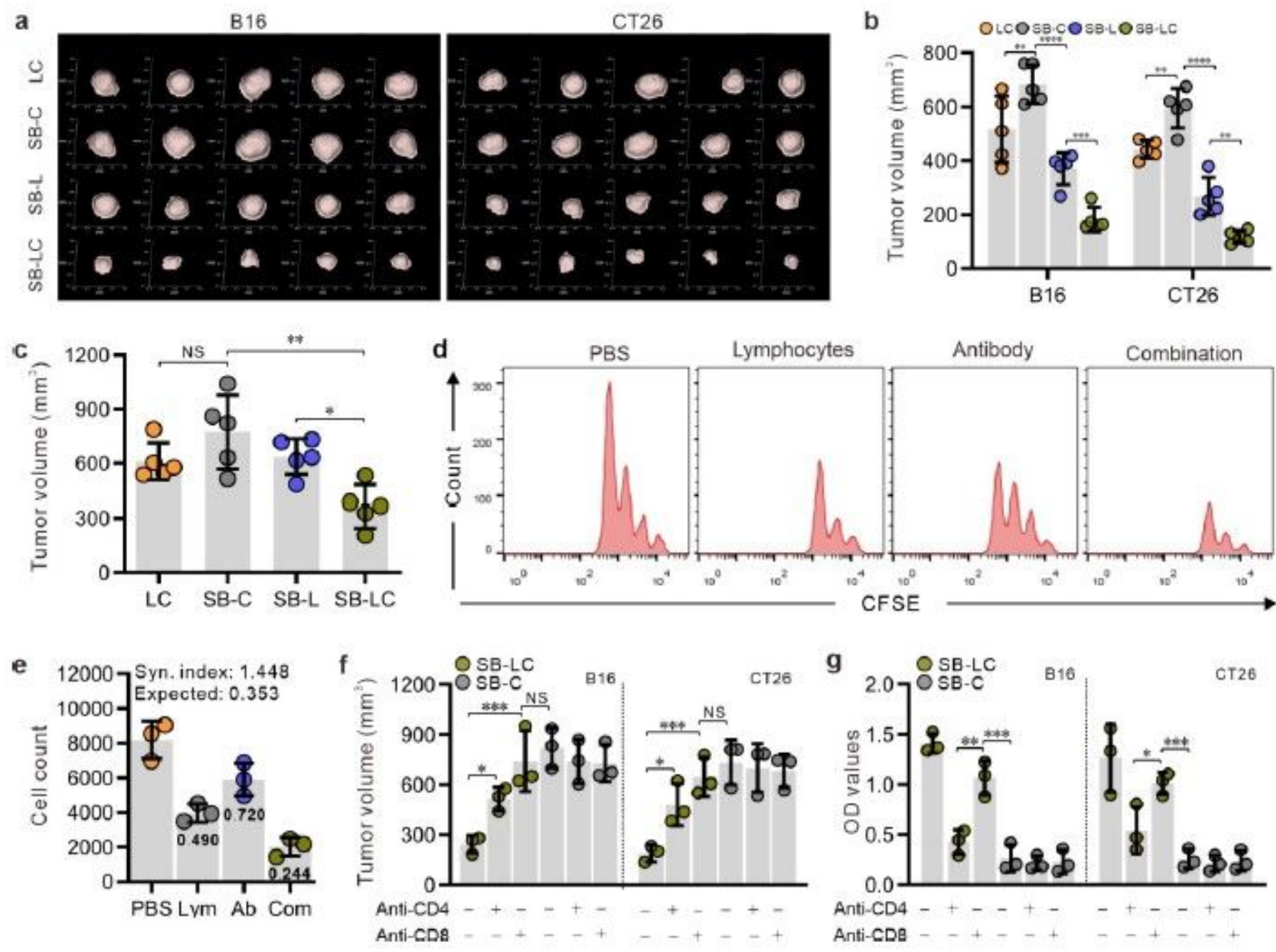

\section{Figure 5}

Cellular and Humoral Immunities Act Synergistically to Inhibit Tumor Growth (A and B) The images showing tumour masses of B16-bearing mice (A), and the tumour volume of B16- and CT26-bearing mice 
(B) on day 15 after tumour cell inoculation and adaptive transfer of splenocytes from mice vaccinated with the indicated formulations (mean $\pm S D, n=5$ ). (C) The tumour volume of B16-bearing mice on day 15 after tumour cell inoculation and adaptive transfer of serum antibodies from mice vaccinated with the indicated formulations (mean $\pm S D, n=5$ ). ( $D$ and E) CFSE-labelled $H 22$ cells injected into the abdominal cavity of BALB/c mice and treated with splenocytes and antibodies from mice vaccinated with SB-LC. Flow cytometric analysis of CD45- H22 cell proliferation (D) and the syngeneic index (E). (F and G) CD4+ or CD8+ cells depleted by intraperitoneal injection of the indicated monoclonal antibodies in B16- or CT26-bearing mice. The tumour growth $(F)$ monitored by a handheld device and the optical density (OD) of the $\lg G$ antibody was detected by ELISA are recorded on day $15(\mathrm{G})$ (mean $\pm S D, n=3$ ). Two- $(B, F$ and G) or one-way (C) ANOVA with Tukey multiple comparisons: ${ }^{*}<0.05, * \star<0.01, * \star \star<0.001, * \star \star *<0.0001$. 

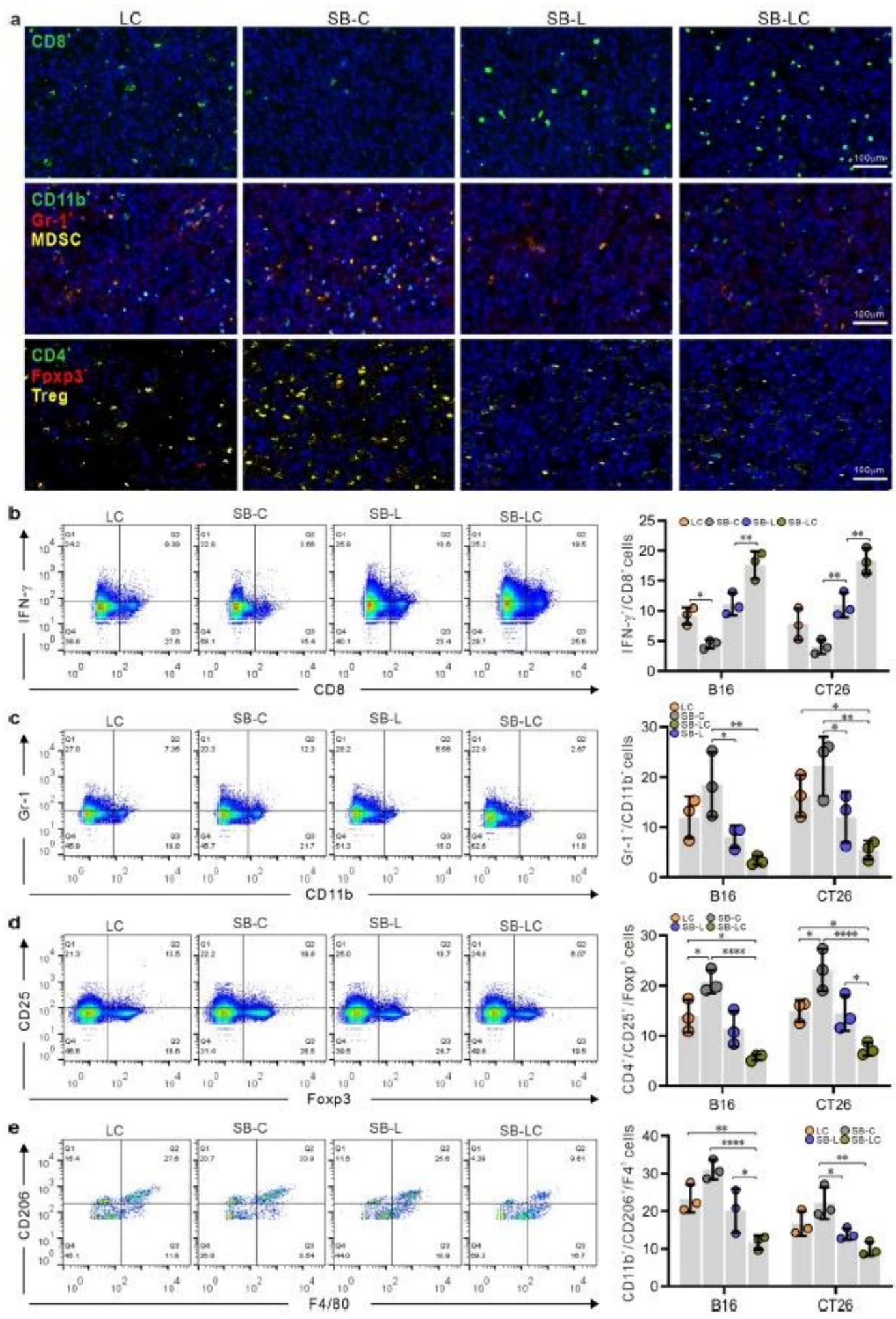

\section{Figure 6}

SB-LC Vaccination Refreshes the Immunosuppressive Tumour Microenvironment. (A) Tumor sections showing CD8+ cells (upper), MDSCs (middle), and Tregs (bottom). (B-E) CD45+CD4+ and CD45+CD4TILs and CD45- tumour cells were gated from the total cell population. Representative flow cytometry images and the mean percentage of TILs with the following features in the B16- or CT26-bearing mice vaccinated with the indicated formulations: IFN-y-secreting CD8+ lymphocytes (B), MDSCs (C), Tregs (D), 
and M2-type tumour-associated macrophages $(E)$ (mean $\pm S D, n=3$ ). Two-way ANOVA with Tukey

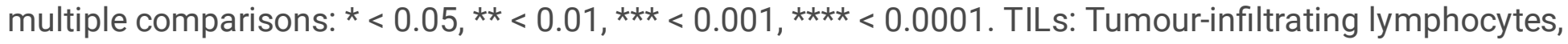
MDSCs: Bone-marrow-derived dendritic cells, Tregs: Regulatory T cells. See also Figure S4.

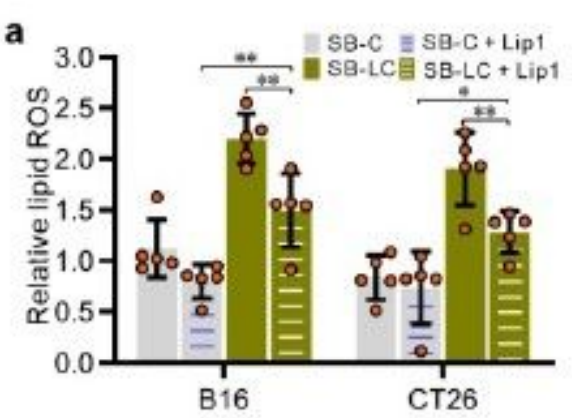

d
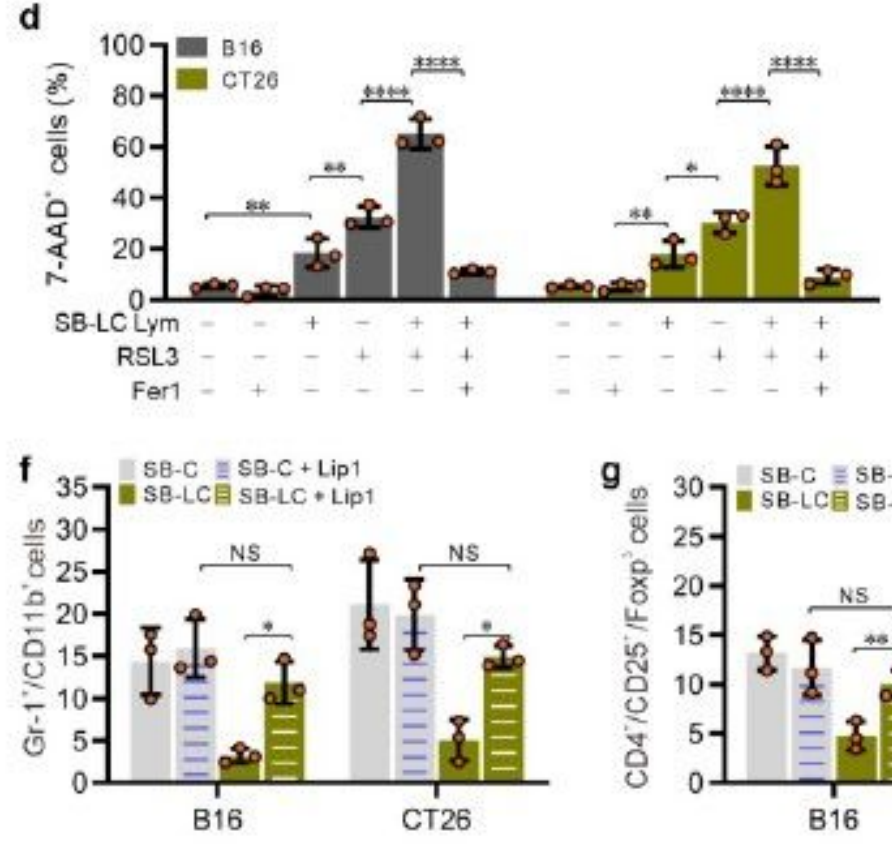
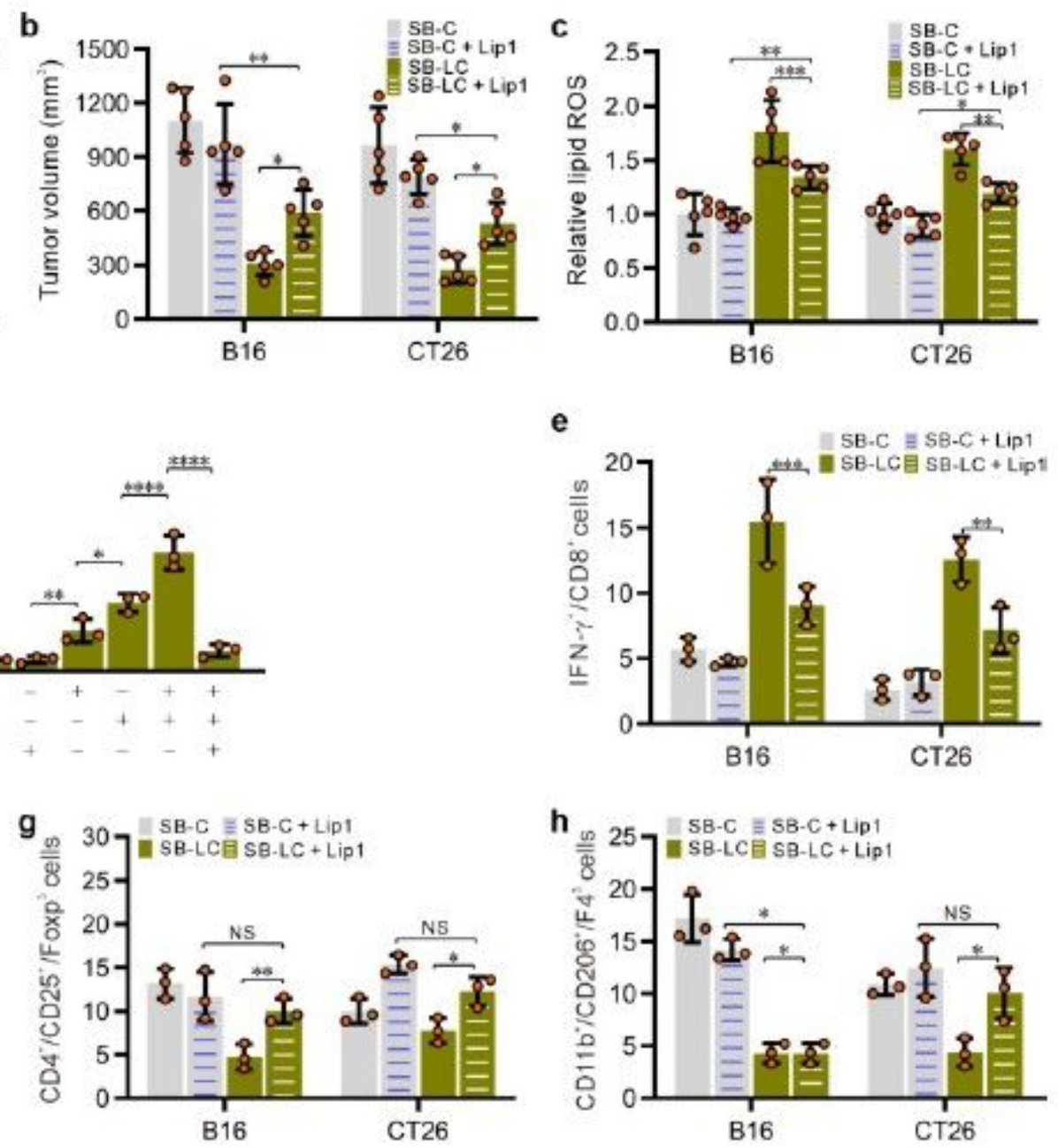

\section{Figure 7}

CTL-Induced Tumour Ferroptosis Reverses the Immunosuppressive Tumour Microenvironment ( $A$ and $B$ ) Relative lipid ROS of the CD45- cells (A) and tumour growth on day 15 after tumour cell inoculation (B) in B16- and CT26-bearing mice vaccinated with the indicated formulations. (C and D) Relative lipid ROS (C) and the percentage of 7-ADD+ (dead) cells among the CD45- B16 or CT26 cells co-cultured with CD45+ splenocytes from mice vaccinated with the indicated formulations. $(E-H) C D 45+$ with or without $C D 4+$ splenocytes from mice vaccinated with the indicated formulations were gated from the co-cultured CD45- B16 or CT26 cells. The mean percentage of the CD45+ splenocytes with the following features is shown: IFN- $y$-secreting CD8+ lymphocytes (E), MDSCs (F), Tregs (G), and M2-type tumour-associated macrophages $(H)$. Data are shown as the mean $\pm S D, n=3$. Two-way ANOVA with Tukey multiple comparisons: ${ }^{*}<0.05, * \star<0.01, \star \star \star<0.001, * \star \star \star<0.0001$. See also Figures S5-S7. 


\section{Supplementary Files}

This is a list of supplementary files associated with this preprint. Click to download.

- SupplementalFigures.pdf 